\title{
43 Natural Anticancer Products: Classified under the Cancer Hallmarks and the Available Evidence of their Anticancer Activities
}

\author{
Dwight L. McKee ${ }^{1, *}$ and Mamoona S. Lodhi \\ ${ }^{1}$ Integrative Cancer Consulting, Aptos, CA, USA; ${ }^{2}$ Mamji Orthopedic \& General Hospital, Karachi, Pakistan
}

\begin{abstract}
About $60 \%$ of chemotherapeutic agents used for the treatment of cancer diseases today have been derived from natural products. While some of these agents are identical to the natural molecules found in plants; the others are semisynthetic derivative of the foundational molecule found naturally in the raw sources. Cancers have been reported to express 10 specific hallmark which are used as the key points or steps for targeted therapy against these cancers. Extending the number of these hallmarks to 12 this review article throws light on 43 natural products classifying them according to their target of action. Further, the natural products under consideration are categorized according to the level of evidence present for their anticancer activities.
\end{abstract}

Keywords: Chemotherapy, anticancer activity, meta-analysis, nutritional supplements, stilbene, invasion, metastasis, cancer stem cells.

\section{INTRODUCTION}

Approximately $60 \%$ of existing chemotherapy agents originate from natural products. Some of these are identical to the parent molecule found in the plant such as vincristine; in other cases, the final drug is a semisynthetic derivative of the foundational molecule found in the natural source. 5 major cancer drugs of marine origin including cytarabine, vidarabine, trabectedin, eribulin mesylate, bleomycin, and brentuximab vedotin have been approved [1].

In addition, the widely used chemotherapy agents irinotecan, topotecan, vincristine, vinblastine, etoposide, and paclitaxel are plant derived whereas, actinomycin $D$ and mitomycin-C originated from bacteria [2].

Apart from these, there are thousands of natural products for which preclinical models have indicated evidence of anticancer activity. Out of this large pool, this review paper discusses 43 natural products, most of them previously used as nutritional supplements, and organizes them according to their activity within the hallmarks of cancer, as originally put forth in 2000 by Hanahan and Weinberg [3,].

The original 6 Hallmarks as published by Hanahan and Weinberg in 2000 were expanded to 10 in their 20111 publication. [4] I have further expanded them in this review to 12 . These 12 cancer hallmarks include: \#1,genomic instability \#2,sustained proliferative

*Address correspondence to this author at the Integrative Cancer Consulting, Aptos, CA, USA; E-mail: dmmdnew@gmail.com signaling \#3,evasion of antigrowth signaling \#4, resistance to apoptosis, \#5, replicative immortality,\#6 dysregulated metabolism, \#7 tumor promoting inflammation, \#8 immune system evasion, \#9 formation of a tumor microenvironment \#10 neo-angiogenesis \#11 tissue invasion and metastasis and \#12 presence of cancer stem cells.

Besides organizing these natural products according to the above mentioned hallmarks of cancer, they are further arranged into the following 8 categories regarding the status of evidence: \#1 evidence of cancer preventive activity in humans; \#2 presence of anecdotal clinical evidence of efficacy in some human cancer type; \#3 presence of epidemiologic evidence of cancer prevention and/or anticancer activity in human cancer; \#4 presence of nonrandomized clinical trials in human cancers with evidence of anticancer activity; \#5 presence of randomized clinical trials in human cancers with evidence of anticancer activity; \#6 presence of meta-analysis of randomized clinical trials looking at cancer death endpoints\#7 clinical evidence of reduction of side effects of conventional cancer therapy/improved tolerance to cancer therapy; and \#8 presence of preclinical--in vitro and/or in vivo--activity against human cancer models but absence of published evidence of clinical anti-cancer activity in humans.

\section{Hallmark \#1: Genomic Instability}

There are three stilbene compounds, all of which share antioxidant activity and the potential to stabilize genomic instability within cancer cells. Of these three: resveratrol, pterostilbine, and piceatannol; resveratrol is the best known although with the least bioavailability. 
Several strategies can be used to increase resveratrol's bioavailability including forming it into nano emulsions, encapsulating it within cyclodextrin molecules, as well as "micronizing" it [5].

All 3 of these stillbenes are found in a wide variety of plants including grapes, peanuts, and berries. Stilbene compounds provide plants with resistance to microbial and fungal infection and therefore plant sources of these compounds have higher levels of these protective compounds if grown without pesticides and herbicides.

The root of Japanese knotweed is the richest known source of resveratrol; pure trans-resveratrol (as well as pterostilbene and piceatannol) can also be produced in bio-reactors in high purity by fermentation. Because cancer cells' signaling networks are extraordinarily complex, the therapeutic success of specific inhibitors has been limited. though natural products often have weak effects, they simultaneously address dozens of targets; and when combined with other natural products having a similarly broad spectrum targeting of multiple signaling pathways, there is potential for clinically significant beneficial effects, especially when given in high bioavailability forms, and significant amounts.

Resveratrol blocks nuclear factor kappa Beta (NF$\mathrm{kB})$, a signaling pathway which regulates inflammation, cellular response to stimuli, and immune response to infection [6].

Resveratrol can inhibit the phosphatidylinositol 3kinase (Pi3K/Akt) pathway which regulates cell growth differentiation and proliferation. It has also been shown to significantly inhibit the IGF-1R/Akt/Wnt pathways and activate p53, thereby influencing tumorigenesis and cell development. Resveratrol also possesses inhibitory activity of the cyclooxygenases which are responsible for the conversion of arachidonic acid into inflammatory prostaglandins [6].

Clinical trials in cancer of nonpatentable natural products are in general scarce, primarily due to the lack of funding. Evidence for activity of resveratrol against cancer is in category \#4 (indication from nonrandomized clinical trials in human cancer with evidence of anticancer activities). Note: clinical trial of resveratrol in myeloma patients showed an increased incidence of renal failure, the mechanism of which is not yet understood. Therefore, stilbenes should NOT be used in experimental or integrative treatment of human patients with multiple myeloma [7].
The other two mentioned stilbenes (pterostilbene and piceatannol), are also classified under the first hallmark (genomic instability), but their evidence for anticancer activity in humans is in category 8 (indicates preclinical in vitro and/or in vivo activity against human cancer models but no clinical evidence of anticancer activity in humans) $[8,9]$.

Vitamin $D$ is also known for its effect to repair the genomic defects during oncogenesis [10].

It is included in anti-cancer activity category \#5 (indicates randomized clinical trials in human cancer with evidence of anticancer activity) vitamin D supplementation with vitamin D3 (but not with vitamin D2)has been reported to cause a significant reduction in cancer mortality, with a risk ratio of 0.85 , indicating that vitamin $\mathrm{D}$ supplementation with a dose greater than $2000 \mathrm{IU} /$ day was associated with a $15 \%$ reduction in mortality from cancer [11].

Selenium is another micronutrient known to improve genomic instability. Many preclinical studies show that selenium enriched yeast is a particularly effective vehicle for supplying this micronutrient $[12,13]$.

Evidences for selenium's activity against cancer are found for both categories 1 (cancer preventive activity in humans), and 5 (randomized clinical trial evidence in human cancer with evidence of anti-cancer activity). One of the most impressive clinical trials on selenium was carried out in 2007 that used sodium selenite in $\mathbf{5 0}$ patients with newly diagnosed non-Hodgkin's lymphoma, randomized in two groups, one of which received Se along with chemotherapy, while the other group received the same chemotherapy alone [14].

In this study, supplementation with Sodium selenite for 30 days in addition to standard chemotherapy was shown to result in a significant decline of the BCL-2 level of lymphoma cells compared to the same chemotherapy alone. In addition, complete responses (CRs) in the selenium added chemotherapy group were $60 \%$, versus $40 \%$ in the chemotherapy alone group. It was also noted that the CD4/CD8 ratio was increased in the selenium group as was overall survival time $(p=0.01)$

Another natural product in the category of improving intrinsic genomic instability in cancer is delta tocotrienol, one of the 8 members of the vitamin $E$ complex. Tocotrienols are smaller than tocopherols, unsaturated, and have a shorter half life ( $4 \mathrm{hrs}$ vs 20 hrs). it is the smallest member of the vitamin E family, 
and has the shortest 'tail', giving it more mobility between lipid bilayers within cell membranes. It has the strongest effect of the four tocotrienols on the inhibition of nuclear factor kappa $B$, as well as on shortening pancreatic cancer cell survival. In vitro strong activity against pancreatic cancer stem-like cells has also been observed for this member [15].

Delta tocotrienol is placed in clinical category 4, indicating nonrandomized clinical trials in human cancer with evidence of anticancer activity.

A phase 2 trial of delta tocotrienol showed that the addition of this member of the vitamin $E$ family, in a dose of $300 \mathrm{mg} 3$ times daily along with IV bevacizumab as maintenance therapy given every 3 weeks to 23 patients with refractory ovarian cancer resulted in a much longer progression free and overall survival when compared with controls, receiving maintenance therapy only with IV bevacizumab [16].

In another phase 1 clinical trial where 200 to 1600 $\mathrm{mg}$ of delta tocotrienol was given daily for 2 weeks prior to surgery for resectable pancreatic cancer it was revealed that it significantly induced apoptosis in pancreatic ductal carcinoma cells and was also well tolerated [17]

The larger tocopherols can block the absorption of the smaller tocotrienols, so it is best to avoid concurrent administration [18].

The seventh natural product in this group (stabilizers of genomic instability) is sulforaphane, a sulphur -containing compound which is a major component of all vegetables within the cruciferous family. Higher dietary intake of cruciferous vegetables has been associated with lower incidence of many forms of cancer especially aggressive forms of prostate cancer, where it has been shown to inhibit androgen receptor signaling [19].

The clinical category for its anticancer activity is category \#4 (non randomized clinical trials in human cancer with evidence of anticancer activity). This was shown in a phase 2 clinical trial of sulforaphane rich broccoli sprouts extracts in men with recurrent prostate cancer. In this clinical trial 20 men with recurrent prostate cancer were given $200 \mu \mathrm{mol} /$ day of sulforaphane as a standardized broccoli sprout preparation. Although only 1 patient experienced a greater than or equal to $50 \%$ PSA decline (the primary endpoint), there was a significant lengthening of the PSA doubling time whilst taking the sulforaphane extract from 6.1 months prior to treatment versus 9.6 months PSADT on treatment $(p=0.044)$. Because the treatment was so well-tolerated and the increase in PSA doubling time on treatment was statistically significant, it was suggested that further studies with higher doses over longer periods of time may be warranted to gain further knowledge regarding the role of sulforaphane as a preventive and/or treatment agent for prostate cancer as well as other cancer types [19].

In a feasibility trial using 15 capsules/day of dried broccoli sprouts providing $7620 \mu \mathrm{mol}$ sulforaphane in stage IV pancreatic cancer patients receiving no other treatment, there was a significantly longer survival percentage over the first year in the treatment group, but an over $70 \%$ dropout rate after the first 6 months. Some patients in the treatment group reported increased $\mathrm{Gl}$ distress related to the intake of 15 capsules/day of dried broccoli sprouts [20].

The final natural product compound that exhibits particularly strong activity against the genomic instability hallmark is the non-provitamin A carotenoid, known as lycopene.

Although dietary intake and tissue levels of provitamin $A$ and related carotenoids have been associated with reduced risk of several types of cancer and many of the same carotenoids have shown anticancer activity in preclinical models, both in vitro and in vivo, intervention trials with isolated carotenoid supplements other than lycopene have not shown anticancer activity, and in fact $1 \%$ of current and former smokers as well as patients with a history of asbestos exposure who took long-term high daily doses of isolated carotenoid supplements actually developed lung cancer which similar patients in the control group did not [21].

Numerous preclinical models, both in vitro and in vivo, have shown activity of lycopene against many human tumor types [22]. Clinical evidence for activity of lycopene against human cancer is category \#3 (epidemiologic evidence of cancer prevention and/or anticancer activity in human cancer). One clinical trial which showed evidence of anticancer activity for lycopene against human prostate cancer was a randomized two arm trial in which patients who had been recently diagnosed with prostate cancer and were scheduled to undergo radical prostatectomy were randomly assigned to either no intervention or $30 \mathrm{mg}$ of daily lycopene supplementation for 3 weeks prior to surgery. It was reported that the PSA level decreased by $18 \%$ in the intervention group whereas it increased 
by $14 \%$ in the control group over the 3-week preoperative study period. Also significant was the observation that within the lycopene supplemented group, 11 of 15 patients $(73 \%)$ had no tumor involvement of surgical margins and/or evidence of extra-prostatic tissues with tumor, whereas only 2 of 11 patients $(18 \%)$ in the control group fulfilled these criteria. Regarding tumor size, 12 of 15 patients (80\%) in the lycopene supplemented group had tumor size that measured $4 \mathrm{cc}$ or less, as compared to only 5 of $11(45 \%)$ in the non-supplemented control [22].

\section{Hallmark \#2: Sustained Proliferative Signaling}

The second of Hanahan and Weinberg's Hallmarks of Cancer, sustained proliferative signaling, is achieved by tumors in several ways. First, tumor cells may produce growth factor ligands to which they respond, they can also signal normal cells in the tumor microenvironment which then supply the cancer cells with growth signals, thus making them no longer dependent on external signals such as epidermal growth factor. Sustained proliferation signaling depends largely on 3 particular signaling pathways: Akt, mTOR, and MAPK [23].

EGCG (epigallocatechin gallate) is one of the major catechins in green tea, and appears to be responsible for much of the anti-cancer activity of green tea. Both EGCG, green tea and green tea extract have been studied for their cancer prevention, anti-cancer stem cell activity, and enhancement of cancer therapeutics for 40 years, particularly in Japan.

Clinical categorization of this natural compound places it in category \#1 (cancer preventive activity in humans) and category \#3 (epidemiologic evidence of cancer prevention and/or anticancer activity in human cancer).

Many epidemiologic observation studies in Japan have shown evidence of primary cancer prevention activity of green tea [24].

In one observational study focusing on the recurrence rate of breast cancer in relation to daily consumption of green tea, it was found that out of 390 patients treated previously for stages 1 and 2 breast cancer the recurrence rate for those who drank less than 4 cups of green tea per day was $24.3 \%$ versus $16.7 \%$ for those who drank more than 5 cups. Time to relapse averaged 2.8 years for those who consumed less than 4 cups and was 3.6 years for those who consumed more than 5 cups. For stage III which involved 82 patients the recurrence rate for those drinking less than 4 cups/day was $58.5 \%$ whereas those consuming over 5 cups/day had a recurrence rate of $48.8 \%$.

In another 10 year prospective observational study of 8,552 people over age 40, 419 cases of cancer developed during the 10 year period of observation. In women, those who consumed over 10 cups of green tea $(1200 \mathrm{ml})$ per day this occurrence was 7.3 years later than those who consumed $<3$ cups per day. In males, this occurrence among the two respective groups was 3.2 years later in age, the difference between the two genders is thought to be due to the heavier use of tobacco by males. This study also showed a significant prevention of lung cancer (relative risk.33) in both sexes who consumed $>10$ cups vs $<3$ cups of green tea per day [24].

A pilot study using green tea extract tablets for prevention of metachronous colorectal adenomas was published in 2008. Patient recruited to the study had previously had colorectal adenomas removed by endoscopic polypectomy and 1 year later were confirmed by colonoscopy to be free of recurrence. These patients were then randomized into 2 groups, both maintaining their usual lifestyle and amounts of green tea drinking. The intervention group received 1.5 $\mathrm{g}$ of green tea extract as tablets (equivalent to about 2 cups of Japanese green tea). All patients were examined by colonoscopy 1 year later and the green tea extract tablet supplement group had a $15 \%$ recurrence rate (9 of 60 ) versus a recurrence rate in the control group of $31 \%$ (20 of 65 ). Relative risk was $0.49 ; 95 \%$ confidence interval, 0.24-0.99; $\mathrm{P}<0.05$. The size of relapsed adenomas was also smaller in the GTE supplemented group than in the control group ( $P$ $<0.001$ ) [25].

In addition, EGCG has been shown in the laboratory to decrease the expression of mRNAs and proteins that serve as stemness markers of Human Cancer Stem Cells [24].

Second of the natural products addressing sustained proliferative signaling is curcumin, a major component of the spice turmeric, long used in Indian cuisine and in Ayurvedic medicine for thousands of years.

Curcumin's clinical category is \#6, which indicates meta-analysis of randomized clinical trials looking at cancer death endpoints. It has been given safely in doses up to $8 \mathrm{~g} /$ day taken orally for 3 months. It has 
shown evidence of activity against oral, lung, and bladder cancers, uterine and cervical intraepithelial neoplasms, oral leukoplakia, intestinal metaplasia of the stomach, colorectal, pancreatic, breast, prostate, head and neck cancers, and multiple myeloma. Although evidence of activity in different types of malignancies has been noted in various types of clinical trials, well-designed randomized placebo controlled trials in various cancer types would be necessary to fully document this [26].

Due to the curcuminoids' limited bioavailability it would be desirable to use them in either a nanoemulsion or liposomal form, combined with piperine, a compound from black pepper which inhibits small intestinal 3A4 and p-glycoprotein activity and can increase the persistence of blood levels of curcuminoids up to 2000 fold. In vitro, curcumin has also demonstrated anticancer stem cell activity [27].

The third of the natural products that primarily address the sustained proliferative signaling of neoplastic conditions, is the isoflavone genistein, which makes up approximately $50 \%$ of all the isoflavones found in soybeans. The isoflavones are also very weak estrogens (about $1 / 1000^{\text {th }}$ the potency of estradiol), which has led to them also being known as 'phytoestrogens'. These compounds bind to the beta estrogen receptor much more potently than to the alpha estrogen receptor, which makes them particularly interesting in hormonally responsive cancers such as breast and prostate, as well as in thyroid and colorectal cancers which express ER beta [28].

The clinical classification of genistein puts it in categories \#1 (cancer preventive activity in humans) \#3 (epidemiologic evidence of cancer prevention and/or anticancer activity in human cancer) and \#4 (indicates non-randomized clinical trials in human cancer with evidence of anticancer activity).

In a placebo-controlled block-randomized doubleblind phase 2 clinical trial, men with localized prostate cancer were randomized to either $30 \mathrm{mg}$ of synthetic genistein taken daily for 3 to 6 weeks prior to prostatectomy, or to placebo. In this trial serum PSA decreased by $7.8 \%$ in the genistein arm whereas they increased by $4.4 \%$ in the placebo arm $(P=0.051)$. There were no significant effects on sex or thyroid hormones and total cholesterol was significantly lower in the genistein arm $(P=0.013)$. The authors noted that genistein at a dose that can be easily obtained from a diet rich in soy products reduced serum PSA in patients with localized prostate cancer without any effects on hormones, was well-tolerated, and had a beneficial effect on blood cholesterol [29].

In a study of over 500 women followed for 5 years after initial breast cancer treatment, it was noted that among postmenopausal patients with ER/PR positive breast cancer who were receiving anastrozole as endocrine therapy, those in the highest quartile of soy isoflavone dietary intake had a significantly lower rate of breast cancer recurrence than those in the lowest quartile $(P=0.02)[30]$.

As part of a complex natural product supplement that addresses all the known Hallmarks of Cancer, in highly bioavailable forms, suggested intake of genistein would be $50 \mathrm{mg}$ twice daily, a dose which is achievable within the range of diets rich in soy foods, and which has been well tolerated and has shown to be therapeutically active in human clinical trials [31].

\section{Hallmark \#3: Evasion of antigrowth signaling}

Hallmarks \#2 and \#3 together allow malignant cells to undergo continuous proliferation. Antigrowth signaling is carried out primarily by tumor suppressor proteins. Retinoblastoma was the first identified tumor suppressor protein (1971) that functions to suppress the growth unless it becomes phosphorylated, a process which turns off its antigrowth signaling. Of the other mutated tumor suppressor genes (resulting in dysfunctional tumor suppressor proteins) the most frequently mutated one is p53. Other key tumor suppressor genes/proteins (in order of frequency of mutation) include PTEN, APC, ATM, BRCA2, VHL, CDKN2A, BRCA1 and WT1. Aside from gene mutation or loss (through chromosomal breaks or translocations), another mechanism which helps tumor cells evade antigrowth signaling is epigenetic silencing of the tumor suppressor genes, which includes histone methylation and acetylation, as well as DNA methylation. Epigenetic silencing of tumor suppressor genes is potentially reversible and many natural compounds can target the restoration of tumor suppressors, which raises the possibility of enhancing cancer prevention and therapy through restoration of functional tumor suppressor proteins with consequent reactivation of anti-growth signaling [32].

Natural products to be discussed in relation to Hallmark \#3 include luteolin and melatonin. As mentioned previously, many natural products are active in relation to multiple hallmarks of cancer and previously discussed compounds including EGCG, curcumin, genistein and resveratrol, have also shown activity against Hallmark \#3 [32]. 
Luteolin $(3,4,5,7$-tetra hydroxy flavone) is a flavonoid found in many plants including edible vegetables, fruits and medicinal herbs. It has poor oral bioavailability, and so formulation into nano particle crystals with sodium dodecyl sulfate, nanoemulsions, or implanted into cyclodextrin molecules should be explored to optimize absorption and pharmacokinetics [33].

Luteolin's clinical evidence categorization is \#8 (indicates preclinical--in vitro and/or in vivo--activity against human cancer models but absence of published evidence of clinical anti-cancer activity in humans).

In Traditional Chinese Medicine (TCM), plants rich in luteolin are used in the treatment of hypertension, inflammatory disorders, and cancer. Although it has not yet been used in human clinical trials, it's preclinical activity both in vitro and in vivo models is impressive. It inhibits the proliferation of tumor cells, and activates cell cycle arrest, in addition it can reverse epithelialmesenchymal transition (EMT), a necessary step in the metastatic process of tumors.

In doses ranging between 1.2 and $40 \mathrm{mg} / \mathrm{kg}$ luteolin has shown impressive anticancer activity both in vitro and in vivo in human models of breast cancer, colon cancer, pancreatic cancer, prostate cancer, glioblastoma, oral, lung, kidney, cervical and placental cancer, ovarian, skin, liver, gastric, esophageal, and bladder cancer, with no evidence of toxicity [34].

Melatonin (N-acetyl-5-methoxy-tryptamine) is a chronotropic hormone that is primarily synthesized in the pineal gland but also in other parts of the body including skin, lymphocytes, eyes, bone marrow, and the gastrointestinal tract. Melatonin is a hormone with autocrine, paracrine, and potent antioxidant effects which exerts diverse receptor independent and receptor dependent actions with pleiotropic effects and overall homeostatic activity relevant to cell protection and survival. It is also known to possess immunomodulating, antiproliferative, and oncostatic effects [35].

Melatonin's clinical evidence categorization is \#5 (indicates randomized clinical trials in human cancer with evidence of anticancer activity), \#6 (indicates meta-analysis of randomized clinical trials looking at cancer death endpoints) and \#7 (indicates clinical evidence of reduction of side effects of conventional cancer therapy).
In addition to melatonin's effect on inhibiting the ability of cancer cells to evade antigrowth signaling it is active against several other Hallmarks including resistance to apoptosis, immune system evasion, and angiogenesis. Part of melatonin's antiproliferative effect appears to be related to its ability to inhibit uptake of linoleic acid by tumor cells through inhibition of cyclic AMP production. In preclinical models melatonin has been shown to have oncostatic properties in a wide variety of tumors including cervical, breast, ovarian, skin, laryngeal, colorectal and prostate cancer, as well as sarcoma, hepatocarcinoma, melanoma, neural tumors and laryngeal carcinoma.

Melatonin also has antiestrogenic properties related to its ability to reduce the expression of ER alpha and inhibit the binding of the estradiol/estrogen receptor complex to the estrogen response element on DNA. This antiestrogen effect very likely contributes to melatonin's" oncostatic activity in estrogen-dependent tumors such as ER- positive breast cancer. There have been many clinical trials done with melatonin, almost always given at a dose of $20 \mathrm{mg}$ in the evening, with thousands of patients both in combination with radiation and, and also tested versus palliative care as well as with low-dose IL-2 in advanced cancer patients. Virtually all of these clinical trials show statistically significant improvement not only in complete and partial remission rates; 1,2 , and 5-year survival rates; but also in tolerance to conventional therapy reducing occurrences of alopecia, anemia, asthenia, and thrombocytopenia [35].

\section{Hallmark \#4-Resistance to Apoptosis}

Apoptosis is the term used to describe "programmed cell death", which is a normal physiological process of all cells. Cancer cells however, lose their ability to undergo apoptosis thus leading to uncontrolled proliferation. Cancer cells often express many of the proteins which are central to resist the activation of the apoptotic cascade. Many of today's mainstream cancer therapies including chemotherapy, radiotherapy, targeted agents, and even some forms of immunotherapy destroy cancer cells primarily by triggering apoptosis [32].

Apigenin (4',5,7,trihdroxy flavone) belongs to the flavone subclass and is abundant in vegetables and fruits such as parsley, grapes, apples and chamomile tea. It is also one of the active ingredients in many Chinese medicinal herbs. It is poorly soluble in water. Various strategies have been used to increase bioavailability such as nano emulsion/suspension, 
polymeric micelles, and liposomes. Animal studies with blood kinetics analysis of radiolabeled apigenin showed a maximum distribution of radioactivity at 24 hours post ingestion time suggesting a slow distribution phase and slow elimination, leading to the possibility that apigenin may accumulate over time if ingested daily in a highly bioavailable form [36].

Apigenin's clinical evidence categorization is \#8 (indicates preclinical--in vitro and/or in vivo--activity against human cancer models but absence of published evidence of clinical anti-cancer activity in humans). One human clinical trial to investigate the adjuvant effect of apigenin combined with quercetin in resected colorectal cancer patients has been designed in Dresden Germany, but has not been started.

Although apigenin shows high antioxidative activity in normal cells, one of the ways it can induce an apoptotic cell death is by increasing the generation of reactive oxygen species within cancer cells. It also shows the capacity to down regulate antiapoptotic factors such as $B C L-2$ and $B C L-X L$ as well as to up regulate the pro-apoptotic factors- Bax and Bim. Apigenin can also induce cell cycle arrest at the G2/M and $S$ phases, in cancer cells in vitro; in addition It can suppress metastasis of cancer cells by interfering with the PI3K/Akt/mTOR signaling pathways, as well as interfering with the expression of MMP-9, one of the key matrix metalloproteinases needed by cancer cells to invade connective tissues as part of the metastatic process [37].

Fisetin $\left(3,3^{\prime}, 4^{\prime}, 7\right.$-tetrahydroxyflavone $)$ is a dietary flavonoid quite similar to apigenin (in fact, fisetin is one of the apigenin metabolites). Preclinical studies have shown that fisetin inhibits cancer growth by inducing apoptosis, altering the cell cycle, inhibiting angiogenesis and inhibiting invasion and metastasis, without causing any toxicity to normal cells. Fisetin binds to and interacts with multiple cellular targets resulting in regulation of a wide variety of functions inside and between malignant cells. It disrupts Wnt signaling resulting in cell cycle arrest, it suppresses the epithelial to mesenchymal transition process thus preventing invasion and migration of cancer cells by inhibiting the YP-1 binding protein. Fisetin physically interacts with the mTOR molecule, thus inhibiting signaling involved in cell survival which explains its inhibitory effect on cellular proliferation. Fisetin binds to micro tubules disrupting their dynamics and acting as a microtubule stabilizing agent superior to paclitaxel. Across all preclinical studies of oral cancer types the most commonly affected signaling pathways by fisetin are mTOR and NF-kB [38].

Fisetin shows strong activity in preclinical models for lung, prostate, colorectal, bladder, breast, and cervical cancers, as well as leukemias (including acute and chronic) and melanoma as well as basal and squamous cell carcinomas of the skin [38].

In terms of the category of evidence for Fisetin, anticancer activity shown in a single randomized clinical trial in human cancer with evidence of anticancer activity places it in category \#5. Because a growing body of evidence indicates that chronic inflammation is associated with tumorigenesis, metastasis, and chemotherapy resistance in different types of cancer, and that naturally occurring flavonoids show anti-inflammatory and antitumor effects in preclinical models, a clinical trial was designed to assess the efficacy of supplementation with fisetin on the inflammatory status and matrix metallo proteinase levels in patients undergoing chemotherapy for colorectal cancer. A double-blind randomized placebo controlled clinical trial with 37 colorectal cancer patients undergoing chemotherapy was designed and carried out. Patients were assigned to receive either $100 \mathrm{mg}$ fisetin or placebo for 7 consecutive weeks. Supplementation began 1 week before chemotherapy commenced and continued until the end of the second chemotherapy cycle. At the conclusion of the study it was noted the plasma levels of interleukin-8 $(P<.04)$ and high sensitivity $C$-reactive protein $(P<.01)$ were reduced significantly in the fisetin group, indicating an anti-inflammatory effect. In addition it was noted that matrix metallic proteinase 7 levels were also significantly reduced in the supplemented group $(P<$ 0.02 ), which would be consistent with an antimetastatic effect of supplementation with fisetin [39].

In terms of safety, fisetin showed no evidence of toxicity in animal models given at $2000 \mathrm{mg} / \mathrm{kg}$ body weight for 48 hours, nor were there any indications of toxicity in any organs using standard toxicological pathology criteria in a long-term feeding in animals fed $25 \mathrm{mg} / \mathrm{kg}$ body weight for 9 months. It was negative in the Ames test, showed no inhibition of hERG activity at concentrations up to 10 micromolar and also showed no inhibitory effects on the activities of hepatic cytochrome P450 enzymes 3A4, 2C9, and 2D6 [40].

Fisetin has been widely available as a dietary supplement since 2018, with people routinely taking $500 \mathrm{mg}$ to 5 grams daily, with no reports of toxicity. 
Di-indole-methane (DIM) is a plant derived indole from cruciferous family of vegetables. Several clinical trials have been performed with a form of DIM which has been micro encapsulated and provided in an extended release form to enhance bioavailability and sustain its effects in the body.

DIM's clinical category is \#4 indicating nonrandomized clinical trials in human cancer with evidence of anticancer activity.

Clinical trials with DIM have focused on prostate and cervical cancer. In patients with castrate resistant prostate cancer (CRPG) who experienced progression after androgen deprivation therapy, DIM administered in clinical trials was well-tolerated and prostate biopsies showed that $93 \%$ of patients receiving DIM had detectable prostatic DIM levels. Also observed in clinical trials with DIM were inhibitory effects on the androgen receptor and androgen receptor target genes including PSA. Clinical trials have been proposed in CRPC patients treated with enzalutamide in combination with DIM.

In one clinical trial with 78 women of reproductive age diagnosed with cervical intraepithelial neoplasia (CIN) stages I and II, patients were randomized to either $200 \mathrm{mg}$ DIM, $100 \mathrm{mg}$ DIM or placebo, administered in the form of vaginal suppositories containing DIM. After either 90 or 180 days of treatment the $100 \mathrm{mg} /$ day suppository group showed regression of $\mathrm{CIN}$ in $86 \%$ of patients and the 200 $\mathrm{mg} /$ day suppository group showed $95 \%$ regression whereas the placebo group showed $58 \%$ regression-these results were statistically significant [41].

In breast cancer DIM showed both evidence of activity in preclinical models as well as epidemiological evidence for prevention of breast cancer in humans [42].

\section{Hallmark \#5-Replicative Immortality}

The ability to maintain continuous self-renewal is necessary for the propagation of normal germ cells but is not a property of normal somatic cells. Genetic damage and consequent genetic instability in previously normal somatic cells can result in development of replicative immortality. This allows the accumulation of further traumatic changes that confer unregulated cell growth, invasiveness, and resistance to therapeutic interventions. Senescence is part of the normal process by which aged and/or damaged cells are removed or undergo growth arrest which prevents affected cells from proliferating even in the presence of appropriate mitogenic factors. Senescence is also a specific response to the gradual shortening of telomeres- the repeating DNA sequences at the end of chromosomes which become shorter with each cell division and dictate the total number of cell divisions which a cell can undergo. Activation of tumor suppressor genes p53 and p16/pRB can also trigger senescence. Genotoxic drugs used in conventional cancer therapy can often induce cancer cell senescence at much lower doses than those required to induce apoptosis and cell death. Targeted therapies have also been developed which induce senescence in cancer cells by blocking cyclin-dependent kinase mediated inhibition of retinoblastoma (RB) family proteins, or by interfering with the activity of the enzyme telomerase within the tumor microenvironment, which is required by tumor cells to maintain telomere length despite replicative immortality [32].

Genistein, curcumin, and EGCG all have activity that facilitates development of the senescent state in cancer cells, though they have already been discussed in other Hallmarks. D-Limonene is a naturally occurring monoterpene, and is one of the major terpene components of the outer skin of virtually all citrus fruits. Perillyl alcohol, is a related compound with similar activity; oral intake of both results in similar metabolites found in human urine, many of which show activity toward the induction of senescence in cancer cells.

In 1998 the results of a phase 1 clinical trial followed by a limited phase 2 evaluation of breast cancer were published. In this trial a group of 32 patients with refractory solid tumors were given $\mathrm{d}$-limonene in escalating doses from $0.5 \mathrm{mg} / \mathrm{m}^{2}$ up to $12 \mathrm{~g} / \mathrm{m}^{2}$ given orally in 21-day cycles. Pharmacokinetics were assessed by liquid chromatography-mass spectrometry. At the conclusion of the phase 1 dose escalation trial, 10 additional advanced cancer patients received fifteen 21-day cycles of $D$-limonene given at 8 $\mathrm{g} / \mathrm{m}^{2} /$ day. In two patient's intratumoral monoterpene levels were measured. Intratumoral levels of $d$ limonene exceeded the corresponding plasma levels.

In the phase 1 trial, the MTD was $8 \mathrm{~g} / \mathrm{m}^{2} /$ day; diarrhea, nausea, and vomiting were the dose-limiting symptoms. 3 patients with colorectal carcinoma had prolonged stable disease and one partial response in a breast cancer patient was maintained for 11 months. The conclusion of the investigators was that the favorable toxicity profile of $d$-limonene, and evidence of clinical anticancer activity supports further clinical evaluation [43]. 
A case/control observational study amongst an older population in Arizona was published in 2000, which showed that citrus peel in the diet was associated with a significantly reduced risk of squamous cell carcinoma of the skin. Overall risk reduction for squamous cell carcinoma of the skin was $0.66,95 \% \mathrm{Cl}=0.45-0.95$. Citrus peel consumption is the major source of dietary d-limonene [44].

Clinical categorization of d-limonene includes category 3-epidemiologic evidence of cancer prevention; and \#4-nonrandomized clinical trials in human cancer with evidence of anticancer activity.

\section{Hallmark \#6-Dysregulated Metabolism}

This hallmark refers to the dysregulated metabolism of cancer cells, which includes an increased production of lactate and an increased uptake of glucose-- which is further metabolized by fermentation- an inefficient metabolic pathway, without using the mitochondria, resulting in only 2 molecules of ATP per molecule of glucose (versus 36 molecules of ATP per molecule of glucose with mitochondrial metabolism). The overproduction of lactate by this process results in acidification of the tumor microenvironment, which inhibits the function of the host immune system and favors the growth and metabolism of malignant cells. The high level of glucose utilization also generates raw materials that can be recycled into new cancer cells. In addition to glucose, the amino acid glutamine is also used as a fuel by proliferating cancer cells, which provides an additional source of carbon and nitrogen. Because these pathways are crucial to normal cell metabolism under different physiologic conditions, it has been difficult to develop preclinical models that are specific to cancer cell metabolism of glucose and glutamine. However broad-spectrum approaches such as fasting, intermittent fasting, calorie restriction, ketogenic diet (with and without calorie restriction) and exercise, all have the potential of fundamentally influencing cancer metabolism (and very likely many other hallmarks of cancer) in therapeutic ways [32]. To date a number of small pilot studies of short-term fasting with chemotherapy have shown that fasting the day before the day of and the day after chemotherapy (72 hours fasting), is strongly associated with both improved tolerance of chemotherapy and decreased measurable toxicity, including DNA damage and normal cells. There has been no evidence of short-term fasting interfering with the response to chemotherapy. There are as yet no long-term studies to look at clinical outcomes of chemotherapy given with short-term fasting [45].

\section{Hallmark \#7-Tumor Promoting Inflammation}

It has been known for over a century that chronic inflammation is strongly linked with malignancy. An inflammatory milieu within the tumor microenvironment favors the expansion of genomic aberrations as well as both the initiation of carcinogenesis and continued tumor growth and metastasis. Chronic inflammation is linked closely to various phases of cancer development including cell transformation, cellular proliferation, evasion of apoptosis, neoangiogenesis and metastasis. Chronic inflammation influences immune system constituents which ordinarily function as first-line defenders against pathogens under normal conditions: immune cells, including natural killer cells, CD4 and CD8 lymphocytes, dendritic cells, mast cells, leukocytes, and macrophages, When tumor cells begin to multiply within the tissue, macrophages and mast cells produce matrix remodeling proteins, chemokines and cytokines which activate local stromal cells such as fibroblasts, vascular cells, and adipocytes, which are recruited by circulating leukocytes into the "damaged" tissue resulting in acute inflammation. When these processes are initiated within the tumor microenvironment they do not resolve thus leading to chronic inflammation of the "damaged" tumor tissue.

All of these cells of the immune system when recruited into the tumor microenvironment began to secrete inflammatory mediators which serve the needs of the tumor rather than those of the host [32].

A large number of natural products possess inherent anti-inflammatory activity including lycopene, genistein, EGCG, resveratrol, and curcumin all of which have been discussed in previous hallmarks.

Natural products that will be discussed under Hallmark \#7 (tumor promoting inflammation) include anthocyanins, sea cucumber extract, and Boswellia serrataextract

Anthocyanins are plant derived phenolic phytochemicals that contribute dark coloration to many berries and other fruits, vegetables, and beans. They share strong antioxidant activity with the many other members of the flavonoid family.

Clinical categorization of anthocyanins would be category \#4 indicating nonrandomized clinical trials in human cancer with evidence of anticancer activity.

Over 500 types of anthocyanins are known, which can be found in 27 families and 72 genera of plants. Research since the 1980s has focused on extraction, 
isolation, metabolism, bioavailability, purification and absorption, pharmacokinetics, and anti-cancer effects of anthocyanins, both in pre-clinical models, and in some limited clinical trials focused on studying changes in metabolism in cancer patients associated with long term ingestion of anthocyanins [46].

Research in preclinical models with anthocyanins has revealed a multitude of activities which act against tumorigenesis including antioxidant, anti-inflammation, and anti-mutagenic effects. Anthocyanins can control secretion and expression of inflammatory factors from various cells including tumor, immune, and stromal cells within the tumor microenvironment, by inhibiting NF-kB via multiple pathways, including MAPK and $\mathrm{PI} 3 \mathrm{~K} / \mathrm{PKB}$, as well as inhibiting the expression of Cox 2 and inducible NO synthase. It is also been shown that it can block the activation of STAT3, as well as actually inhibiting the expression of NF-kB [46].

In addition to the effect of anthocyanins against the hallmark of 'tumor promoting inflammation' they are also active in other hallmarks of cancer including inducing apoptosis as well as inhibiting angiogenesis in tumors and inhibiting tissue invasion and metastasis of tumors [46].

A phase 1 pilot study in 20 patients with Barrett's esophagus (a premalignant condition with a 30-40 fold increased risk for development of esophageal adenocarcinoma), in which surveys, biopsies, blood, and urine samples were collected before and after 6 months of daily supplementation with lyophilized black raspberries (either 32 or $45 \mathrm{~g}$ ). Immunohistochemical staining of Barrett's esophagus biopsies following 6 months of lyophilized black raspberry supplementation showed a statistically significant increase in expression of GST-pi, a marker of detoxification. It was also noted that lyophilized black raspberry supplementation significantly reduced urinary excretion of 8 epi prostaglandin F2 alpha, a marker of oxidative stress and free radical damage in lipids. In another clinical trial, 28 patients with colorectal cancer patient were given $60 \mathrm{~g}$ of lyophilized black raspberries daily for 1 to 9 weeks, with urine and plasma specimens collected before and after black raspberry supplementation. Mass spectrometry based metabolomic analysis of these specimens, revealed increased levels of 4-methyl catechol sulfate in both urine and plasma specimens of patients after black raspberry supplementation, and this plasma and urine marker correlated with a higher level of TUNEL, a marker of apoptosis, in resected tumors [47].

\section{Sea Cucumber Extract}

Sea cucumbers are invertebrate marine organisms which are nutrient rich and have been used for centuries as an anti-inflammatory food source throughout Asia. They contain the longchain omega-3 fatty acids EPA and DHA, as well as many glycosaminoglycans, including analogs of heparin. Laboratory research in animal models has revealed anti-inflammatory activity 3 times as potent as that of aspirin [48].

In addition to the anti-inflammatory activity of sea cucumber and its extracts antitumor activity has been demonstrated, in other preclinical models, including cell cycle arrest, DNA fragmentation and apoptosis of tumor cells, maturation of dendritic cells to improve immune response, increase in lysosomal activity resulting in increased phagocytosis of macrophages, and antiproliferative activity [48].

Clinical categorization of sea cucumber extract is category \#4-indicates nonrandomized clinical trials in human cancer with evidence of anticancer activity.

In a phase 2 clinical trial of an extract of sea cucumber- supplied and characterized by Coastside Bio resources- known as Frondanol A5 (a water/isopropyl alcohol extract), was given to 15 patients with asymptomatic multiple myeloma with a median follow-up of 21 months. 5 of these patients developed progressive disease while receiving the extract. Supplementation with the Sea cucumber extract was well-tolerated, and further clinical trials were suggested by the authors [49].

\section{Boswellia Serrata Extract (60\% Boswellic Acid)}

Boswellic acids are triterpenes derived from the sap of a tree native to the Middle East and widely known as frankincense. The boswellic acids are well-known natural products which possess anti-inflammatory, antiapoptotic and cytotoxic effects on various cancer cell lines while at the same time being harmless to normal cells. In vitro, Boswellic acids show anticancer effects against 14 different cancer cell lines; in vivo models show anticancer effects for colorectal cancer, Ehrlich tumor, glioma, leukemia, pancreatic cancer, and prostate cancer [50].

The clinical category for Boswellia is \#5, which indicates randomized clinical trials in human cancer with evidence of anticancer activity.

In one clinical trial, 44 patients with secondary or primary malignant brain tumors were randomly 
assigned to radiotherapy along with either daily oral intake of $4.2 \mathrm{~g}$ of whole Boswellia serrata resin, or placebo. Cerebral edema post- radiotherapy was assessed by T2 weighted MRI sequences. Results showed a reduction of cerebral edema of more than $75 \%$ in $60 \%$ of patients receiving the active treatment and in $26 \%$ of patients receiving placebo $(P=0.023)$. An additional antitumor effect in the Boswellia treated group was postulated by the investigators, but could not be documented within the design of the study. Serum levels of patients who had been taking Boswellia resin were positive for boswellic acids [51].

\section{Hallmark \#8-Immune System Evasion}

One of the hallmarks of malignant tumors is that they hide from and evade immune attack by multiple mechanisms. These include secretion of thrombogenic factors which create fibrin layers over and around tumors, generating signals to increase the generation of T-regulatory cells, induction of immune suppressive mediators by tumor cells themselves or by host cells recruited to the tumor microenvironment by the tumor. Other mechanisms of tumor cells include synthesis of decoy proteins which cause $T$ cells to bind and undergo apoptosis, defective antigen presentation, and creating an immunosuppressive environment within the tumor microenvironment, one component of which, is the acid $\mathrm{pH}$ of the tumor microenvironment generated by export of lactic acid from within tumor cells where it is generated by aerobic fermentation of glucose. One of the major advances in cancer immunotherapy within the past decade was the development of the checkpoint inhibitor antibodies which bind to the decoy proteins on tumor cells which would otherwise cause $T$ cells to continually undergo apoptosis in the tumor microenvironment. Other immunotherapy strategies which hold the potential to extend the efficacy of checkpoint inhibitors include reduction of Treg lymphocytes, enhancing natural killer cell activity, activation of macrophages, adoptive T-cell therapy, CAR-T-cell therapy, and newly developing CAR-NK cell therapy, both autologous and allogeneic (so called 'offthe-shelf' CAR-T and CAR NK cells) $[32,52,53]$.

In addition, the tumor microenvironment can be altered via diet, supplementation, exercise, stress management, alkalization, and supplementation with various phytochemicals, to become less immunosuppressive, and thus support systemic cancer immunotherapy [54]. Phytochemicals which may be helpful in working against immune system evasion by tumor cells include:

\section{Reishi (Ganoderma Lucidum)}

Ganoderma lucidum, commonly known as reishi, is one of the "medicinal" mushrooms, used as part of traditional Asian medicine for thousands of years. Its immune activating components are polysaccharides and preclinical studies have established that the polysaccharide fraction of this mushroom has potent antitumor activity, associated with the stimulating effects of these polysaccharides on the immune system.

Clinical categorization for this mushroom is category \#4, indicating nonrandomized clinical trials in human cancer with evidence of anticancer activity.

One study of the polysaccharide fraction extracted from $\mathrm{G}$. lucidum on the immune function of advanced stage cancer patients was published in 2003.

34 advanced stage cancer patients were treated with $1800 \mathrm{mg}$ of the polysaccharide extract three times daily, taken orally prior to meals for 3 months. Of the 34 patients, 30 completed the 12-week trial and were assessable for their immune functions. Significant findings included an increase in the mean plasma concentrations of interleukin-2, interleukin-6, and interferon gamma, whereas the levels of tumor necrosis factor alpha and interleukin-1 were significantly decreased. The mean absolute number of CD56 positive lymphocytes were significantly increased $(P<0.05)$. Phytohemagglutinin $(\mathrm{PHA})$ responses were enhanced in most patients after the 12-week treatment when compared to pretreatment baseline's $(P<0.05)$. Also noted was a significant increase $(P<0.05)$ in the mean natural killer cell activity compared to baseline. All of these immunologic shifts were in the direction of restoration of immune competence, and although not likely by themselves to have a major therapeutic effect in advanced cancer patients, they do suggest that this therapy could be beneficial in concert with immunotherapy of earlier stage cancers and also in helping prevent relapse after treatment [54].

\section{Turkey Tail Mushroom (Trametes Versicolor)}

Previously known as Coriolus versicolor, known as Yun zhi in China and Turkey tail in North America, this colorful mushroom which grows on trees throughout the world has long been used in traditional Asian medical systems and has long been believed to generally promote strength, longevity, and health. The predominant polysaccharide extracts of it are known as PSP (China), and PSK (Japan), where they are both 
currently approved as adjuvant therapy along with chemotherapy or radiotherapy both for gastric cancer and non-small cell lung cancer. A meta-analysis of 13 randomized placebo controlled double-blind clinical trials was published in 2012. The findings showed that treatment with polysaccharides from Trametes versicolor, in patients with breast cancer, gastric cancer, or colorectal cancer treated with chemotherapy had a $9 \%$ absolute reduction in 5-year mortality, resulting in 1 additional patient being alive for every 11 patients treated with the Trametes extracts [55].

Clinical categorization would be \#5, which indicates randomized clinical trials in human cancer with evidence of anticancer activity.

\section{Astragalus Extract (15:1)}

Astragalus membranceus is a woody root very rich in saponins. It has been used for many centuries in traditional Chinese medicine both by itself and in combination with other medicinal herbs. Astragalus's immunologic benefits include activation of M1 macrophages, enhancing natural killer cell activity, inhibiting $\mathrm{T}$ helper cell type 2 cytokines (thereby enhancing Th1/cell-mediated immunity).

Clinical categorization of Astragalus includes category \#4 (none randomized clinical trials in human cancer with evidence of anticancer activity) and \#6 (indicates meta-analysis of randomized clinical trials in human cancer looking at cancer death endpoints.

A meta-analysis of Astragalus-based Chinese herbs and platinum-based chemotherapy for advanced nonsmall cell lung cancer was published in 2006 in the Journal of clinical oncology by McCulloch et al. 34 randomized studies representing 2,815 patients were included in the meta-analysis. This meta-analysis documented reduced risk of death at 12 and 24 months as well as improved tumor response data in the groups randomized to chemotherapy plus Astragalus based Chinese herbs [56].

Similar meta-analyses have been published which reviewed multiple randomized clinical trials using Astragalus based Chinese herbs with chemotherapy versus chemotherapy alone, in hepatocellular carcinoma and also in acute myeloid leukemia $[57,58]$.

\section{Yeast Incorporated Zinc}

Zinc is an essential element in the diet, and necessary for normal immune function. Zinc is integral to many transcription factors and proteins that regulate key cellular functions including apoptosis cell cycle progression DNA replication DNA damage repair in the response to oxidative stress. Zinc plays a pivotal role in host defense against the initiation and promotion of several types of malignancy [59].

Zinc requirement in the human diet is approximately $15 \mathrm{mg} /$ day. Approximately $10 \%$ of the US population consumes less than half the required amount of zinc. The prevalence of zinc deficiency in cancer patients has not been widely studied. However, one metaanalysis published in 2015 of studies of serum and hair zinc levels in breast cancer patients versus controls found a consistently lower level of hair zinc in breast cancer patients [60].

There are many forms of zinc salts use as nutritional supplements including zinc sulfate, gluconate, citrate, acetate and picolinate. Zinc is also available as zinc-enriched yeast, in which high levels of zinc salts are added to yeast culture and the yeast then harvested and dried. It is the most well-tolerated and also shows increased bioavailability compared to other forms of zinc both in human and in animal studies [61, 62].

The clinical categorization for zinc is category \#5 which indicates randomized clinical trials in human cancer with evidence of anticancer activity.

In 2017 a prospective randomized double-blind placebo-controlled trial was published in which 24 patients receiving chemotherapy for colorectal carcinoma were randomized to receive zinc capsules $35 \mathrm{mg}$ each or placebo. The capsules were taken twice daily for 16 weeks from the immediate postoperative period to the fourth chemotherapy cycle. Assessment with the functional assessment of chronic illness therapy (FACT-fatigue) showed that the placebo group developed a worsening quality of life and increased fatigue between the first and fourth chemotherapy cycles whereas there were no changes in the scores of quality of life or fatigue in the zinc supplemented group [63].

In another double-blind placebo controlled clinical trial of 34 patients with stages 3 and 4 nasopharyngeal carcinoma were randomized to receive $75 \mathrm{mg} /$ day of zinc supplements or placebo for 2 months in conjunction with chemoradiation. Patient to receive the zinc supplements showed a higher 5-year overall and disease-free survival rate than the patients in the placebo group $(P<0.05)[64]$. 
Hallmark \#9: Tissue Interactions in the Tumor Microenvironment

Cancer cells and host cells evolve in an in- vivo tumor microenvironment in which both direct and indirect cellular interactions produce metabolites and secrete factors that affect cancer progression or regression. The tumor microenvironment regulates the ability of the cancer to grow and survive via many biological processes including inflammation, relative hypoxia and $\mathrm{pH}$, with lower levels of oxygen and lower $\mathrm{pH}$, and higher levels of inflammation favoring tumor growth. The innate and adaptive immune system is also active within the tumor microenvironment which can be either immunosuppressive, favoring cancer growth, or immune activating, favoring stability or regression of tumor. Host microenvironment processes such as fibrosis and angiogenesis as well as cytokine mediated regulatory programs involving interleukin-6, endoglin, JAK, and many others, can also influence the balance between tumor and host cells within the tumor microenvironment [32]. Many aspects of diet, lifestyle, micronutrients, and phytonutrients can impact many of these processes within the tumor microenvironment [54].

Berberine, an alkaloid constituent in a number of medicinal herbs has been long used in traditional Chinese medicine, Ayurveda, and other traditional Asian medical systems to treat infections and inflammatory disorders. Within the tumor microenvironment tumor cells secrete cytokines which promote tumor cell proliferation and metastasis. Berberine has extensive anticancer effects documented in preclinical models of 21 cancer types. In an osteosarcoma model berberine showed a strong anti-inflammatory effect in the tumor microenvironment related to potent down-regulation of the caspase-1/IL1 beta signaling pathway [65].

It also has been shown to suppress the T cells' response via direct interaction with dendritic and $T$ cells. Thus berberine regulates the tumor microenvironment both by affecting immune cells, their secretions, and by inhibiting the inflammatory response necessary for tumor progression.

In addition to its anticancer modulating activity of the tumor microenvironment, berberine has been shown to be active in a wide variety of anticancer mechanisms including the inhibition of tumor cell proliferation related to regulation of tumor cell autophagy as well as regulation of the cell cycle, and also through the promotion of tumor cell apoptosis.
Although berberine possesses strong antioxidant activity in normal cells, within tumor cells it has been shown to be capable of inducing oxidative DNA damage while simultaneously down regulating homologous recombination repair of DNA within tumor cells, an effect that synergizes with PARP inhibitors [66].

Berberine inhibits tumor cell invasion and metastasis by down regulating metastasis related proteins and signaling pathways related to the epithelial-mesenchymal transition necessary to initiate the metastatic process. It also inhibits tumor cell proliferation by suppressing telomerase activity and by interacting with micro-RNAs necessary for tumor cell proliferation. Berberine is currently in clinical trials in China for prevention of colonic adenomas in patients who are S/P resection of colorectal cancers.

Berberine's clinical classification is category \#8, indicating preclinical research evidence of anticancer activity, but no clinical or epidemiological anticancer data yet available.

Berberine is only slightly soluble in water at body temperature with consequent low bioavailability. Various strategies have been developed to overcome this issue including the design of new formulation strategies including nano-sized dosage forms, liposomes, micro-emulsions, nano-emulsions, mucoadhesive microparticles, and phospholipid complexes [67].

Zerumbone is a natural compound isolated from the roots of Zingiber zerumbet (commonly known as 'ginger'), traditionally used as an appetizer and food flavoring agent in many cuisines. It has also long been used in traditional Asian medical systems for relief of inflammation, pain, and nausea and has also been widely used in herbal folk medicine around the world. Like berberine, it is known for it strong antiinflammatory activities as well as being antioxidant, antibacterial, antinociceptive, antipyretic, and antihypertensive, as well as possessing immunomodulatory and hepatoprotective properties [68].

In addition to its strong immunomodulatory and antiinflammatory effects within the tumor microenvironment, in preclinical in- vivo models it also exhibits diverse effects against tumor cell proliferation angiogenesis and promotes tumor cell apoptosis. Preclinical studies show activity of zerumbone against 17 different human cancer types [68]. 
Clinical studies with whole ginger root $(2 \mathrm{~g} /$ day $)$ in people with increased risk of colon cancer have shown decreases in markers of colonic epithelium which suggest a decrease risk for development of colorectal cancer [69].

Clinical categorization of zerumbone is category \#1, indicating evidence for cancer preventive activity in humans.

Due to low bioavailability, zerumbone would best be used in either a nano emulsion formulation or a watersoluble cyclodextrin complex.

\section{Hallmark \#10: Tumor Angiogenesis}

Angiogenesis, which describes the creation of new blood vessels, is essential for tumor development. Micrometastatic deposits of tumors are unable to grow beyond the size of $2-3 \mathrm{~mm}^{3}$ without initiating tumor angiogenesis. Tumor hypoxia which develops when tumor deposits near the size of several cubic millimeters is a potent inducer of tumor angiogenesis. With tumor progression increasing genomic instability within the tumor results and mutations within pathways regulating the production of multiple angiogenic factors, and normal stroma cells recruited to the tumor microenvironment also become significant sources of longer-term angiogenic growth factor production. This complex angiogenic growth factor profile argues against the use of single monoclonal antibodies directed against angiogenic growth factors such as vascular endothelial growth factor (VEGF), or fibroblast growth factor (FGF) [32].

Phytochemicals are generally well-tolerated in combination over extended periods of time and often demonstrate broader mechanisms of action against key angiogenesis targets. Targeting aspects of the tumor microenvironment which are potent stimuli to angiogenesis can also be useful, such as reducing structural abnormalities within tumor blood vessels, reducing the elevated interstitial fluid pressure, reducing hypoxia, reversing poor tumor perfusion, suppressing tumor promoting inflammation, and deactivating tumor promoting fibroblasts as well as reversing acidosis within the tumor microenvironment, both by blocking lactic acid transport from cancer cells and use of systemic and/or local tumor microenvironment alkalinization [32].

A number of natural products which have potent antiangiogenesis effects on tumors have already been discussed in relationship to other hallmarks, including curcumin, EGCG, resveratrol, and melatonin. Natural products to be discussed specifically in terms of their antiangiogenesis effect include Silibinin (from milk thistle), Baicalein (from Scutellaria baicalesis), Kaempferol, a flavonoid found in many edible botanicals, and Oleanoic acid, an isomer of ursolic acid, sourced from olive leaves.

Silibinin (and its parent flavonoid compound sylimarin) is a flavonoid derived from the milk thistle plant (Silybum marianum) which has potent antioxidant and hepatoprotective activity and ancient medicinal usage. In addition to potent anti-angiogenesis effects in tumors, silibinin has also shown the ability to inhibit tumor cell proliferation, induce apoptosis, modulate cell signaling, and inhibit tissue invasion and metastasis of tumor cells. Strong preclinical evidence of anticancer effects for 17 human cancer types has been documented. In addition it exhibits potent anticancer stem cell effects in vitro [70].

Most clinical trials have focused on and developed evidence for hepatoprotection in advanced hepatoma as well as hepatoprotection from chemotherapy in pediatric leukemia (ALL) chemotherapy $[71,72]$.

In a randomized clinical trial, silymarin and selenium given for 6 months prior to radical prostatectomy was found to significantly reduce tumor markers of lipid metabolism (LDL and total cholesterol) known to be associated with prostate cancer progression. Those who received silymarin and selenium also showed statistically significant improved quality of life scores compared with the those who received placebo [73].

In a randomized double-blind placebo controlled clinical trial published in 2016 silymarin $(420 \mathrm{mg}$ daily in 3 divided doses starting on the first day of radiotherapy and continued for 6 weeks) was found to significantly reduce oral mucositis from radiotherapy of head and neck cancer [74].

Clinical categorization of silybinin's anticancer effects is category \#7 which indicates clinical evidence of reduction of side effects of conventional cancer therapy and improving tolerance to cancer therapy.

Baicalein is 1 of over 50 flavonoids that have been isolated from the root of Scutellaria baicalensis Georgi, and has been found to be the most active constituent of the dried root which has been used in traditional Chinese medicine for many centuries. In addition to potent antiangiogenesis and anti-inflammatory effects on tumors, it also inhibits complexes of cyclins to 
regulate the cell cycle, attenuates mitogen activated protein kinase (MAPK), protein kinase B (Akt), scavenger's oxidative radicals and also blocks signaling of mTOR, which induces apoptosis. It also inhibits tumor invasion and metastasis by reducing the expression of matrix metallic proteinase-2/-9 [75]. This multi-targeted flavone shows strong preclinical data against many human tumor types. In a phase 1 trial in healthy volunteers low bioavailability was seen, but was dramatically improved by coadministration with piperine (potent inhibitor of CYP-450 3A4), and by nano emulsion. It has only been used in clinical trials as a component of a traditional Chinese medicine formula that has been studied at Yale University school of medicine under the name of PHY906, which is made up of Glycyrrhiza uralensis, Paeonia lactifora, Scutellaria baicalensis, and Ziziphus jujuba. PHY906 has been shown to protect against the severe diarrhea from irinotecan therapy and also has been trialed in a nonrandomized phase 2 trial with capecitabine as second line therapy for pancreatic cancer patients who have progressed on gemcitabine, though clinical results have not yet been published [76].

In addition it has been shown to enhance the oral bioavailability and prolong the half-life of flavonoid silybin via inhibition of efflux transporters BCRP and MRP2 [77].

\section{Kaempferol (3,5,7,4' Tetrahydroxyflavone)}

Kaempferol is found in many edible plants and in various plant parts including flowers, fruits, leaves, seeds, as well as the edible parts of vegetables. It is particularly rich in its non-glycosylated form (the aglycone) in bee pollen, cruciferous vegetables, capers, Chia seeds, cumin, chives, moringa leaves, fennel, garlic, and endive. It is particularly rich in the seeds of the tea plant (Camellia sinensis), which is extracted by super critical CO2/ethanol as a commercial source of this flavonoid.

Kaempferol and its glycosylated derivatives have been shown to have anticancer activities as well as to be cardioprotective, anti-inflammatory, antioxidant, antimicrobial, antidiabetic, and neuroprotective.

Inhibition of angiogenesis is a prominent mechanism of kaempferol's anticancer activity as is its ability to trigger apoptosis in cancer cells, antiproliferative effects related to down-regulation of multiple signaling pathways including $\mathrm{PI} 3 \mathrm{~K} /$ protein kinase $B$, cell cycle arrest at the $G 2 / M$ phase, and inhibition of the epithelial-mesenchymal transition process which is necessary to the metastatic process [78].

Like many flavonoids, kaempferol shows low bioavailability however combining it with quercetin increases its bioavailability; studies with nano formulations of kaempferol have dramatically improved bioavailability with improved efficacy and selectivity for mutated cells. With levels in vivo achieved through nano- formulations, it triggers apoptosis in mutated cells while exerting protective effects in normal cellswhich is related at least in part to the remarkable antioxidant effects within normal cells while having the reverse effect of increasing oxidative stress within malignant cells. Epidemiological studies have revealed that high dietary intakes of kaempferol are associated with a decreased incidence of many different types of cancer including those of bladder, stomach, pancreas, ovary, liver, colon, and skin [78].

Clinical categorization of kaempferol would be category \#3, indicating epidemiologic evidence of cancer prevention and/or anticancer activity in humans.

Oleanolic acid (3-beta-hydroxyolean-12-en-28-oic acid) is one of the most common pentacyclic triterpenoid compounds, found in over 1600 plants. Its primary commercial source is olive leaves. It is a registered drug in China for treatment of liver diseases and for hepatoprotection. Oleanolic acid has the potential to be employed as an adjuvant anticancer treatment in clinical practice. It has direct anticancer activity and can act synergistically with existing chemotherapeutic drugs; it also inhibits reflux transporters thus increasing intracellular concentration of chemotherapeutic drugs in tumor cells. It exhibits a radiosensitizing effect thus increasing the efficacy of radiation therapy and lastly it has very low toxicity and does not have any adverse effects when combined with chemotherapeutic agents at least in preclinical models. Anticancer properties of oleanolic in preclinical models is in the low micromolar range which corresponds to serum values noted in humans eating an oleanolic enriched diet. Nano emulsion forms of this triterpenoid compound will dramatically improve bioavailability according to preclinical models.

Clinical categorization would be category \#8 which is extensive and promising preclinical model evidence but no published evidence of clinical trials in humans.

\section{Hallmark \#11-Tissue Invasion and Metastasis}

It is the process of metastasis that makes cancer a lethal disease. The exceptions are germ cell tumors 
and acute lymphomas which can be cured even in the metastatic setting with current therapies, but make up only about $3 \%$ of total cancers. Most cancer deaths are due to progressive metastatic disease, though some cases of 'oligometastatic' cancer (only a few sites) can remain stable for many years [79].

In order for a tumor to become metastatic, cancer cells within it must go through a series of discrete changes to successfully progress through the metastatic cascade, leading to the acquisition of an invasive phenotype. This process is often referred to as the epithelial-mesenchymal transition (EMT), which enables carcinoma cells to suppress their epithelial features and acquire those of mesenchymal cells, thus allowing the acquisition of mobility and the capacity to migrate from the primary site. This involves loss of cellcell contact with the surrounding tumor and normal host cells within the tumor microenvironment, degradation of the surrounding extracellular matrix, invasion and migration through the surrounding tissue, secretion of angiogenic/lymphangiogenic factors, intravasation to blood/lymph vessels, transport around the body while evading the immune system, extravasation at a secondary site and ultimately establishment of a secondary (metastatic) tumor [80].

Factors associated with metastases such as disruption of E-cadherin and tight junctions, suppression of matrix metallic proteinase activity, and down-regulation of key signaling pathways are currently targets of intensive research, and many natural products exhibit the capacity to do this when studied in preclinical models [80].

Several natural products already mentioned in this review with respect to other cancer hallmarks including Ganoderma lucidum (Reishi), and silibinin from Silybum marianum (milk thistle) possess activity against multiple targets within this hallmark. Other natural products with activity against tissue invasion and metastasis include the omega-3 fatty acids eicosapentaenoic acid (EPA) and Docosohexanenoic acid (DHA), the omega 6 fatty acid, gamma linolenic acid (GLA), the carotenoid astaxanthin, and oligomeric proanthocyanidins (OPCs), and are discussed below.

\section{Gamma Linolenic Acid (GLA)}

Gamma linolenic acid is the only omega 6 fatty acid which has an anti-inflammatory effect due to its conversion to prostaglandin E1, one of the major antiinflammatory prostaglandins. GLA upon absorption from the intestine is rapidly converted to dihomo-GLA (DGLA) which is incorporated into phospholipids of cell membranes where it can be released by the action of the enzyme phospholipase A2. DGLA competes with arachidonic acid for the enzymes cyclooxygenase (COX) and lipoxogenase (LOX). Arachidonic acid is an omega- 6 fatty acid which is rich in animal foods, and one of the most proinflammatory fatty acids. When DGLA is metabolized by cyclooxygenase its products are prostaglandin E1, which is strongly antiinflammatory, and thromboxane A1, which exerts both anti-aggregation (platelets) and vasodilatory actions [81].

Gamma linolenic acid occurs in a number of edible oils including evening primrose oil, borage oil, black currant seed oil and hemp seed oil. In 1995 Jiang demonstrated that in vitro culture with GLA of many types of human tumor cells including breast, melanoma, liver, and lung, enhances the expression of E-cadherin, which is a key antimetastatic factor [82].

Even in tumors that do not express E-cadherin, GLA increases cell adhesion mediated by the desmosomes, thus increasing anti-metastatic effects [83].

In 1995 Das et al. showed that administration of GLA via a cerebral reservoir placed in the tumor bed at a rate of $1 \mathrm{mg} /$ day for 10 days induced regression of cerebral gliomas, as evaluated by CT imaging, and increased survival of the patient's by 1.5 to 2 years over historical controls [84].

Besides the anti-metastatic effect of GLA (increasing expression of E-cadherin, and enhancing cell adhesion mediated by desmosomes), anti-cancer effects of GLA shown in preclinical research include antiangiogenic action and tumor cells stimulation of apoptosis in tumor cells and direct cytotoxic action in tumor cells as well as the anti-inflammatory effects of prostaglandin E1 which is enhanced by GLA [85].

Supplemental GLA has been administered safely in clinical trials of oral doses up to $2.8 \mathrm{~g} /$ day for up to a year with no apparent toxicities or side effects [86].

\section{EPA+GLA}

One concern related to DGLA (metabolite of GLA) is that it can be further metabolized to the proinflammatory arachidonic acid. However, coingesting similar levels of GLA and the omega-3 fatty acid, EPA increases the cellular membrane content of both DGLA and EPA (the latter also being a precursor to anti-inflammatory prostaglandins). This supplementation strategy maintains the anti- 
inflammatory capacity of GLA while increasing serum EPA, without causing accumulation of arachidonic acid.

\section{EPA and DHA (from Fish Oil or Algaes)}

Eicosapentaenoic acid (EPA) and docosahexaenoic acid (DHA) are the 2 major longchain omega-3 fatty acids obtained from Coldwater fish. They also can be extracted from specific algae species. Diets of indigenous peoples living in the arctic were found to contain as high as 15 to $18 \mathrm{~g}$ of these omega- 3 fatty acids per day and yet despite this very high fat diet cancer and cardiovascular disease was quite rare when these populations were first studied by Western scientists several generations ago. Supplements of fish oil have become quite popular and high-quality longchain omega- 3 fatty acids can be quite useful in people who eat fish and other seafood rarely. The dietary omega 6 to omega-3 ratio that our distant ancestors evolved on was in the range of 1-1 up to 4-1, however people in developed countries eating typical western diets have an omega 6 to omega- 3 ratio somewhere between 20-1 and as high as $50-1$, which strongly predisposes to chronic inflammatory conditions.

As early as 1994, mouse models implanted with human breast cancer cells, compared isocaloric diets composed of $23 \%$ total fat, comparing corn oil with fish oil and found that fish oil supplementation was associated with significant suppression of both primary tumor growth rate and lung metastasis occurrence and severity, as compared with corn oil-containing diets in mice [87].

The same authors also investigated tumor responses to EPA or DHA administered immediately after surgical excision of a murine primary tumor; it was found that DHA but not EPA significantly reduced lung metastasis in the post excision period. Similar results were obtained with purified DHA and a murine mammary metastatic tumor model [88].

Several different experimental models of colon cancer metastasis have also been used to study the antimetastatic property of DHA. It was shown that dietary EPA/DHA inhibited both growth and pulmonary colonization of the transplantable tumor in mice. Other investigators using the same subcutaneously implanted highly metastatic colon carcinoma model found that a DHA rich diet dramatically suppressed lung metastases; more significantly, they found that in vivo DHA treated tumor cells maintained their low potential for metastatic lung colony formation when transferred to a new host suggesting that the effect of DHA was exerted directly on the metastatic ability of the tumor cells themselves and not via the microenvironment [89].

DHA in particular has been found to augment the efficacy of chemotherapy agents in preclinical models [90].

On the basis of this, a phase 2 clinical trial of 25 patients with metastatic breast cancer evaluated the addition of $1.8 \mathrm{~g} \mathrm{DHA}$ daily added to an anthracyclinebased chemotherapy regimen. Patients were divided into 2 groups based on high or low DHA incorporation into plasma phospholipids (even though all patients receive the same dose of $\mathrm{DHA}$ ). The group with high incorporation of DHA had a significantly longer time to disease progression (8.7 months versus 3.5 months) and overall survival (34 months versus 18 months). This study suggests that degree of incorporation of DHA and plasma phospholipids may be useful as a marker for dose escalation of DHA to improve clinical efficacy [91].

Several molecular mechanisms have been proposed for these well-documented antimetastatic effects of longchain omega-3 fatty acids and particularly DHA. These include alterations of tumor membrane characteristics induced by uptake of DHA which displaces arachidonic acid from the phospholipid content of tumor cell membranes which may make tumor cell membranes less fluid and less deformable. A favored postulate is that the antimetastatic activity of DHA may be related to changes in the fatty acid composition of tumor cells, impairing tumor cell membrane fluidity and decreasing its ability to metastasize. It is also been observed that increased representation of DHA in tumor cell membrane phospholipids which is associated with a statistically significant reduction in arachidonic acid, is associated with decreased prostaglandin E2 biosynthesis. PGE2 production is key to suppression of immune responses to cancer cells and it promotes inflammation as well as enhancement of tumor cell proliferation, invasion, and tumor angiogenesis. It has also been demonstrated that increased dietary DHA causes a decrease in metalloprotease-9 (MMP-9) in tumor cells, as well as increasing specific endogenous inhibitors of matrix metallic proteinases, which are necessary enzymes for tumors to use in their metastatic cascade. Animal model research also shows that dietary management with the both EPA and DHA significantly inhibits the invasion of tumor cells through the extracellular matrix via multiple mechanisms [92]. 
Multiple clinical trials of fish oil supplementation concurrent with chemotherapy has been shown to increase time to tumor progression (colorectal cancer), has been shown to provide a benefit over standard of care for weight and skeletal muscle mass in patients with non-small cell lung cancer receiving chemotherapy, and has shown increases in first-line chemotherapy efficacy in patients with advanced nonsmall cell lung cancer. Supplementation with EPA has shown improved tolerance of chemotherapy in cancer patients without overt malnutrition [93-96].

Clinical categorization of omega-3 fatty acids and GLA include category \#1, indicating cancer preventive activity in humans, category \#4, indicating. randomized clinical trials in human cancer with evidence of anticancer activity, category \#7, indicating clinical evidence of reduction of side effects of conventional cancer therapy and improvement of tolerance to cancer therapy with reduced side effects.

\section{Oligomeric Proanthocyanidins (OPC)}

Oligomeric proanthocyanidins, abbreviated as OPCs, are a class of phenolic compounds that take the form of oligomers or polymers of polyhydroxy flavan-3ol units such as catechin and epicatechin. OPCs are found widely in the diet; particularly rich sources include black currant, cranberry, blueberry, green and black tea and chocolate. Commercial sources used for extracting and purifying OPCs are primarily pine bark and grapeseed. Polymers of catechin and epicatechin of more than 10 repeating monomers are referred to as tannins and have very low bioavailability.

Experiments with carbon 14 labeled OPCs done both in animals and in humans reveal high bioavailability of the oligomers, and wide distribution throughout the body with evidence for binding to collagen; it is however not yet clear whether these radiolabeled signals represented the original OPCs or metabolites of it. This class of compounds has been shown to possess potent antioxidant effects both in vitro and in vivo, and modulate Nrf2-mediated gene transcription; they also demonstrate potent antiinflammatory effects in vitro and in vivo, at least in part due to down-regulation of the nuclear factor kappa beta (NFk-B) signaling pathway $[97,98]$.

The anticancer effects of OPCs are also related to the down-regulation of NFk-B signaling (antiinflammatory) along with down-regulation of mitogen activated protein kinases, PI3K/Akt, cytokines, caspases, cell cycle regulatory proteins, and angiogenesis. OPCs also specifically inhibit tissue invasion and metastasis via specific molecular targets. In vitro studies with colorectal cancer cell lines confirmed the inhibition of tumor cell migration and modulation of the EMT-associated genes. In addition, OPCs affect pathways implicated in cancer cell motility including axon guidance, focal adhesion, and regulation of actin [99].

Clinical classification of OPCs is category \#8, indicating strong preclinical model evidence of anticancer activity but no published human clinical trial data.

\section{Astaxanthin}

Astaxanthin is one of the most common carotenoids and has a red color; it is a potent antioxidant, and is widely distributed in marine life; it is responsible for the pink color of salmon and multiple crustaceans. Commercially it is extracted from specific algae (haematococcus pluvialis) which are grown either in outdoor ponds or in large bio-reactors. This, and several related algae are the primary source of Astaxanthin in the marine food chain.

In a high throughput drug screening based on invadopodia staining, Astaxanthin was selected as one of the most active compounds with anti-invasive and anti-migratory activity of tumor cells in vitro. Invadopodia are F-actin rich protrusions of the cell membrane which degrade the extracellular matrix and enable invasion of tumor cells through it. Astaxanthin increases miR-200a and miR-29a-3p, which are signaling RNA molecules that suppress the expression of matrix metallic proteinase 2 (MMP2) and ZEB1, which are crucial to the epithelial-mesenchymal transition (EMT) of malignant cells and is necessary for the metastatic process. Astaxanthin was also shown to have antimetastatic activity through the repression of the MYC transcription factor. In addition, it has been shown that Astaxanthin, tested in a human colon cancer mouse model, suppresses in vivo metastatic capacity [100].

In an in vivo animal model of human breast cancer, Astaxanthin was found to significantly reduce tumor cell proliferation rates as well as inhibit breast cancer cell migration [101].

In terms of bioavailability of Astaxanthin, a single dose of $40 \mathrm{mg}$ administered to healthy male volunteers either as a commercially available food supplement or as a lipid-based formulation containing longchain triglycerides or glycerol mono-and dioleate, showed 
that the lipid-based formulations were 1.7-3.7 times more bioavailable than the commercially available food supplement form. In animal studies, nano sized emulsions of Astaxanthin dramatically improved bioavailability and tissue distribution, with the highest concentrations of Astaxanthin found in the spleen, which may explain Astaxanthin's pronounced effect on the immune system. Animal studies concerning concentration of Astaxanthin in organs was shown to be, in descending order: spleen, kidney, heart, lung, and liver [102].

Clinical categorization of Astaxanthin is category \#8, indicating robust preclinical evidence of anticancer activity, with no human clinical trial results in cancer published yet (there have been many clinical trials in other conditions).

\section{Hallmark \#12-Cancer Stem Cells within Tumors}

Cancer stem cells (CSCs) are subpopulations of undifferentiated cancer cells which are highly resistant to radiation and chemotherapy. They are responsible for therapeutic resistance and tumor initiation recurrence. In fact, there is evidence that chemotherapy and radiation can result in increased numbers of cancer stem cells, with evidence suggesting that damaged tumor cells can increase "stemness" in order to survive and persist within the tumor microenvironment [103].

Cancer stem cells also appear to have enhanced abilities in the realm of evasion of immune surveillance. In fact, a growing body of evidence has revealed interplay between CSCs and immune cells within the tumor microenvironment which appear to suggest a role for TME immune cells in driving the stemness of cancer cells as well as enhancing the ability of CSCs to evade immune surveillance. Currently immunotherapy approaches to eliminate CSCs, such as CAR-T cells are designed to recognize CSC cell membrane markers which include CD 24, CD34, CD 44, CD 47, CD 133, and CD 90, although there is also evidence for CSCs without stem cell surface markers [104].

However, expression of such markers has been inconsistent across CSCs from different tumor types and in addition many of the reported CSC markers also occur in healthy stem cells and even in noncancerous non-stem like cells. Importantly, natural products including dietary phytochemicals as naturally occurring compounds with a wide safety profile, have recently gained enormous attention due to their ability to target CSCs by interfering with key signaling and survival pathways of CSCs as demonstrated in experimental, preclinical, and some current clinical studies [105].

Primary signaling mechanisms known to be active in the regulation of CSCs include Janus-activated kinase/signal transducer and activator of transcription (JAK/STAT), nuclear factor-kappa B (NF-kB), phosphatidylnositol 3-kinase (PI3K)/protein kinase B (Akt), notch pathways, Wnt/beta catenin, and hedgehog $(\mathrm{Hh})$ pathway. The value of natural products is a result of their ability to influence multiple signaling pathways simultaneously with usually quite benign side effects. Because virtually all CSCs are multiple drug-resistant, natural products which down regulate multiple drug resistance (MDR) genes are often active against CSCs.

In this review, 8 compounds with anti--CSC activity are already listed under previous hallmarks. These include resveratrol, sulforaphane, EGCG, genistein, curcumin, apigenin, berberine, and silibinin--as noted previously many natural products function against multiple hallmarks of cancer. Complex mixtures of these have never previously been clinically evaluated in cancer.

7 more natural product compounds, each with documented in vitro efficacy against CSCs, all of which also have other anticancer mechanisms are proposed to be added to this mixture, given the critical issue of maximally inhibiting the activity of the CSCs in terms of obtaining and maintaining remission in many types of malignancies.

These 7 include parthenolide, a sequiterpene lactone, which occurs in the flowers of the Feverfew plant (traditionally used for prophylaxis of migraines) in concentrations up to $0.4 \%$. In a high throughput screening of 50,000 compounds for anticancer stem cell activity salinomycin (a microbial derived compound used commercially as an antibiotic in poultry) showed the highest activity, and parthenolide was second [106].

In addition to parthenolide/feverfew, review of the literature suggests including the following 7 natural products:

Myricetin (from Bayberry bark/leaf extract) $100 \mathrm{mg}$ twice daily; quercetin in liposomal form, mixed with bromelain to enhance absorption-- $500 \mathrm{mg}$ Quercetin/100 mg Bromelain twice daily; 6-shogaol (produced from 6-gingerol by heat/dehydration-in nanoemulsion format, $200 \mathrm{mg}$ twice daily; Piperine (purified from black pepper) $20 \mathrm{mg}$ twice daily-in addition to anti-CSC activity, piperine also prolongs the half-lives of curcumin, resveratrol, and silibinin (natural 
products previously covered in this review, all of which possess anti-CSC activity, with each also addressing multiple other hallmarks) and which have short halflives otherwise--piperine also can raise the level of any medication metabolized by CYP3A4, P-glycoprotein, and/or glucuronidation [107].

Honokiol (extracted and purified from magnolia bark), $500 \mathrm{mg}$ twice daily; caffeic acid phenethyl ester (CAPE) is purified from propolis, a resinous product produced by bees from the resin which coats growing buds of trees. It would be preferable to use in the highly bioavailable form—stabilized and solubilized by complexing with gamma cyclodextrin (Cyclo-Chem, Japan)—500 mg twice daily.

Because there are as yet no clinical means for discovering and monitoring cancer stem cells within tumors, all 15 natural products included in this review that possess anti-CSC activity have primarily been studied with in vitro systems.

In vivo models suffer from the same problem as with the clinical situation-although there are methods for isolating and culturing cancer stem cells in research laboratories, they are all laborious and expensive. However, given the very low toxicity of these natural products and the emerging evidence that cancer stem cells are key factors in resistance to therapy of metastatic tumors and also major contributors to relapse in patients with previous complete responses to current cancer therapies, it would seem prudent to maximize activity against CSCs in this complex mixture of natural products.

\section{CONCLUSION AND FUTURE DIRECTIONS}

\section{Potential Clinical Research Scenarios for Study of the Complex Mixture of Natural Products Covered in this Review}

The use of complex mixtures of phytochemicals and other natural products has been associated primarily with integrative medicine, which is an approach to health and healing that "makes use of all appropriate therapeutic and lifestyle approaches, healthcare professionals, and disciplines to achieve optimal health and healing" [32].

A comprehensive integrative medicine intervention for cancer patients typically includes whole food diets and nutrition education, physical activity recommendations and coaching, and mind-body medicine to help manage stress and anxiety, as well as supplementation with phytochemicals and other natural products. It would be of interest to develop clinical trials for cancer patients at high risk of relapse in which patients would be randomized to a group which would receive a comprehensive integrative medicine intervention along with supplementation of the described complex mixture of natural products, compared with a similar group that would be given no instruction concerning diet, exercise and mind-body medicine, using only supplementation with this complex mixture of natural products. If a large enough group of otherwise relatively homogeneous cancer patients could be recruited, it would be interesting to have 2 placebo groups: one that received a comprehensive integrative medicine intervention and the other which did not, and both groups would receive placebo designed to look similar to tablets of the complex mixture of natural products.

Hallmarks that may be particularly impacted by a comprehensive integrative medicine intervention include tumor promoting inflammation, genomic instability, immune system evasion, and dysregulated metabolism. Dietary interventions in clinical trials have been shown to reduce recurrence risk, reduce inflammation as measured by $\mathrm{C}$-reactive protein, and result in reductions of cytokines and signaling molecules related to tumor progression. Mind-body interventions have been shown in interventional trials to result in activation of $\mathrm{T}$ cells and lymphokine activated killer cells, as well as increases in natural killer cell activity. Exercise interventions have shown documented effects on both progression free and overall survival, increased natural killer cell activity, modulation of sex hormones, and reduction of IGF-1 levels [32].

Preliminary studies of this complex mixture of orally available natural products could involve pharmacologic studies of various components of this mixture; ideally this would be done with the natural products known to be of limited bioavailability by the oral route in humans and/or animals.

Research published by Chamberlin et. al. from Oregon Health and Science University in Portland Oregon, studied known signaling pathways targeted by natural products and compared them with the target networks of conventional chemotherapy agents. They found an increase of $61 \%$ (725 pathways) covered by natural products as compared to FDA approved cancer drugs. Not only did the coverage of pathways targeted by any compound increase when natural products were included, but coverage of targets within those pathways was also increased. The group found 24 pathways 
related to cancer drivers that had no available chemotherapy drugs at the potential for clinically relevant binding affinity threshold of less than $100 \mathrm{nM}$, but for each of these 24 pathways they found at least 1 natural product which addressed that pathway in the same concentration range (less than $100 \mathrm{nM}$ ). Their work highlighted the fact that natural products show groupings of families of targets that are both distinct from and shared by chemotherapy agents-- which strengthens the possibilities for complementary activity of natural products with conventional chemotherapy (and/or immunotherapy, and/or radiotherapy) [108].

There has been very little study of the actual effect of natural product supplementation on the pharmacology of chemotherapy and immunotherapy agents, as well as on their clinical efficacy. Such a study would ideally use patients as their own control, since there is so much inter-individual variation in cancer drug levels due to genetically determined drug metabolizing and elimination enzymes and pathways [109].

One way to do this would be to study cancer patients receiving a regimen of conventional cancer therapy with monitoring of drug levels, and then receiving the same regimen of therapy with monitoring of drug levels while receiving this complex mixture of natural products twice daily. This would involve monitoring peak and trough levels of IV and/or oral chemotherapy and/or immunotherapy agents alone, and then again when combined with the described complex mixture of orally active natural products. This would, of course, be limited to cancer drugs with concentration in serum that can be accurately determined in a clinical or research laboratory.

Although human clinical trials in cancer with phytochemicals and natural products are limited in comparison to those with conventional cancer medicines, many dozens of them have been completed or are ongoing, and preliminary results appear promising. Most of the studies done in the United States have included a single phytochemicals or single herb extract and very rarely were more than 3 or 4 natural products used together. This desire to limit the number of substances studied is at least partially driven by the goal of knowing "what works". However, given the complex nature, resiliency, and metabolic redundancy that research has revealed to be present in most malignancies, complex interventions are more likely to be successful than are targeted ones. In contrast to the Western reductionist approach, a voluminous number of trials (over 3000 of Traditional Chinese Medicine alone) both alone and in combination with conventional cancer treatment have been published. $90 \%$ of these studies used TCM botanical combinations consisting of 8-12 different herbs, each of which addresses many dozens of targets and signaling pathways--and often multiple traditional formulas each consisting of up to a dozen herbs have been used in such trials. However only $16 \%$ of TCM trials were randomized and only a very small percentage were blinded [32]. It would be quite innovative and useful to study the clinical effects of a complex mixture of over 40 natural products, each of which addresses multiple hallmarks of cancer and the mixture of which addresses all the known hallmarks of cancer in a redundant fashion in the context of highquality double-blind randomized placebo controlled clinical trials. Since the role of diet, physical activity, and mind-body therapies have been shown to impact survival of cancer patients [32], these areas represent uncontrolled variables in virtually all the clinical cancer research which has been done up to this time. As mentioned previously it would be of interest to study the effect of these integrative medicine interventions in combination with supplementation of this complex oral mixture of natural products and compare it with a similar group of patients who received the complex oral mixture only with no instruction regarding diet, physical activity, and mind-body therapies. In other settings, particularly when the available number of study patients is limited, it would be useful to control for these 3 variables by providing similar education and support to all the patients in the study regarding diet, physical activity and mind-body therapies, along with educating these patients that improvements in these 3 domains have been shown to improve clinical outcomes in a number of different types of cancer.

Surgical therapy of cancer represents an opportunity for innovative study of complex mixtures of phytochemicals and natural products which address all the known hallmarks of cancer in a redundant fashion, such as described in this review. Once phase 1 dose escalation studies of the natural product mixture with pharmacodynamic study of components of the mixture known to have limited bioavailability have been completed, the highest well-tolerated dose of the mixture could be given perioperatively and during the postoperative period during which chemotherapy and/or radiotherapy are usually not given due to concerns regarding impairment of wound healing. The recent availability of highly sensitive markers of minimal residual disease such as circulating tumor cells and 
even more sensitive assays for circulating tumor DNA could be used in concert with such clinical trials in order to monitor efficacy of the natural product mixture in this setting, or lack thereof.

If the net effect of this complex mixture of natural products was found to significantly affect conventional cancer drug blood levels in an unfavorable way, the mixture could still be studied in concert with immunotherapy, since this uses primarily monoclonal antibodies which are not metabolized by the liver and therefore much less susceptible to herb/nutrient/drug interactions.

It would also be of interest to study the effects of this complex mixture of orally active natural products, on patients who have completed conventional cancer therapy and been found to have no evidence of disease (NED) according to clinical criteria such as imaging studies and tumor markers, but have tumor types and/or stages associated with a high risk of recurrence. Such a study could also be done with such NED patients who have not only high risk for recurrence, but also positive blood tests for circulating tumor cells and/or circulating tumor DNA, monitoring of which could prove very useful in evaluating efficacy. In all such clinical trials dietary and lifestyle factors should be addressed and standardized as much as possible, in order to avoid uncontrolled and potentially confounding variables which can affect clinical outcomes.

Clinical trials of this complex mixture of natural products could also be studied concurrently with immunotherapy, such as for metastatic melanoma, and also be continued for long-term after cessation of immunotherapy in a randomized placebo controlled double-blind trial, using both disease-free and overall survival as endpoints, as well as with periodic monitoring of the blood for circulating tumor cells and circulating tumor DNA.

It is possible that such a complex mixture of natural products which addresses all the known hallmarks of cancer, might improve clinical outcomes in several or all of the above discussed clinical scenarios, however only well-designed and well executed double-blind randomized placebo controlled clinical trials will be able to establish whether or not this is the case.

\section{REFERENCES}

[1] Dyshlovoy SA, Honecker F. Marine Compounds and Cancer: The First Two Decades of XXI Century. Mar Drugs. 2019; 18(1): 20

https://doi.org/10.3390/md18010020
[2] Huang M, Lu JJ, Ding J. Natural Products in Cancer Therapy: Past, Present and Future. Nat Prod Bioprospect 2021; 11: 513.

https://doi.org/10.1007/s13659-020-00293-7

[3] Hanahan D, Weinberg RA. The hallmarks of cancer. Cell 2000; 100(1): 57-70.

https://doi.org/10.1016/S0092-8674(00)81683-9

[4] Hanahan D, Weinberg RA. Hallmarks of cancer: the next generation. Cell 2011; 144(5): 646-74. https://doi.org/10.1016/j.cell.2011.02.013

[5] Gean Pablo S. Aguiar, Daiane L. Boschetto, Lorenzo M.P.C. Chaves, Bianca D. Arcari, Angelo L. Piato, J. Vladimir Oliveira, Marcelo Lanza, Trans-resveratrol micronization by SEDS technique. Industrial Crops and Products 2016; 89: 350-355.

https://doi.org/10.1016/j.indcrop.2016.04.047

[6] Kundu, Shin, Kim, et al. resveratrol inhibits phorbol esterinduced expression of Cox 2 and activation of NF-Kappa B in mouse skin by blocking IkappaB kinase activity. Carcinogenesis 2006; 27: 1465-1474. https://doi.org/10.1093/carcin/bgi349

[7] Berman AY, Motechin RA, Wiesenfeld MY, et al. The therapeutic potential of resveratrol: a review of clinical trials. npj Precision Onc 2017; 1: 35.

https://doi.org/10.1038/s41698-017-0038-6

[8] Banik K, Ranaware AM, Harsha C, Nitesh T, Girisa S, Deshpande V, Fan L, Nalawade SP, Sethi G, Kunnumakkara $A B$. Piceatannol: A natural stilbene for the prevention and treatment of cancer. Pharmacol Res 2020; 153: 104635 https://doi.org/10.1016/j.phrs.2020.104635

[9] Obrador E, Salvador-Palmer R, Jihad-Jebbar A, et al. Pterostilbene in Cancer Therapy. Antioxidants (Basel) 2021; 10(3): 492.

https://doi.org/10.3390/antiox10030492

[10] Graziano S, Johnston R, Deng O, Zhang J, Gonzalo S. Vitamin D/vitamin D receptor axis regulates DNA repair during oncogene-induced senescence. Oncogene 2016; 35(41): 5362-5376.

https://doi.org/10.1038/onc.2016.77

[11] Zhang $\mathrm{Y}$, Fang $\mathrm{F}$, Tang J, Jia L, Feng $\mathrm{Y}, \mathrm{Xu} \mathrm{P}$, et al. Association between vitamin $\mathrm{D}$ supplementation and mortality: systematic review and meta-analysis BMJ 2019; 366: 14673. https://doi.org/10.1136/bmj.14673

[12] The use of high-selenium yeast to raise selenium status: how does it measure up? Margaret P. Rayman; British Journal of Nutrition 2004; 92: 557-573.

https://doi.org/10.1079/BJN20041251

[13] El-Bayoumy, Karam, Sinha, Raghu, Richie, John. Forms of Selenium in Cancer Prevention 2015.

[14] Asfour IA, Fayek M, Raouf S, Soliman M, Hegab HM, ElDesoky H, Saleh R, Moussa MA. The impact of high-dose sodium selenite therapy on $\mathrm{Bcl}-2$ expression in adult nonHodgkin's lymphoma patients: correlation with response and survival. Biol Trace Elem Res 2007; 120(1-3): 1-10. https://doi.org/10.1007/s12011-007-0029-5

[15] Husain K, Centeno BA, Coppola D, Trevino J, Sebti SM, Malafa MP. $\delta$-Tocotrienol, a natural form of vitamin E, inhibits pancreatic cancer stem-like cells and prevents pancreatic cancer metastasis. Oncotarget 2017; 8(19): 31554-31567. https://doi.org/10.18632/oncotarget.15767

[16] Thomsen $C B$, Andersen RF, Steffensen KD, Adimi $P$, Jakobsen A. Delta tocotrienol in recurrent ovarian cancer. A phase II trial. Pharmacol Res 2019; 141: 392-396. https://doi.org/10.1016/j.phrs.2019.01.017

[17] Springett GM, Husain K, Neuger A, Centeno B, Chen DT, Hutchinson TZ, Lush RM, Sebti S, Malafa MP. A Phase I Safety, Pharmacokinetic, and Pharmacodynamic Presurgical Trial of Vitamin E $\delta$-tocotrienol in Patients with Pancreatic Ductal Neoplasia. EBioMedicine 2015; 2(12): 1987-95. https://doi.org/10.1016/j.ebiom.2015.11.025 
[18] Drotleff AM, Bohnsack C, Schneider I, Hahn A, Ternes W. Human oral bioavailability and pharmacokinetics of tocotrienols from tocotrienol-rich (tocopherol-low) barley oil and palm oil formulations. Journal of Functional Foods 2014; 7: $150-160$. https://doi.org/10.1016/j.jff.2014.01.001

[19] Alumkal JJ, Slottke R, Schwartzman J, et al. A phase II study of sulforaphane-rich broccoli sprout extracts in men with recurrent prostate cancer. Invest New Drugs 2015; 33(2): 480-489.

https://doi.org/10.1007/s10637-014-0189-z

[20] Lozanovski VJ, Polychronidis G, Gross W, et al. Broccoli sprout supplementation in patients with advanced pancreatic cancer is difficult despite positive effects-results from the POUDER pilot study. Invest New Drugs 2020; 38: 776-784. https://doi.org/10.1007/s10637-019-00826-z

[21] Bohn T, Bonet M, Borel P, Keijer J, Landrier J, Milisav I, Dulińska-Litewka J. Mechanistic aspects of carotenoid health benefits - where are we now? Nutrition Research Reviews 2021; 34(2): 276-302.

https://doi.org/10.1017/S0954422421000147

[22] Kucuk O, Sarkar FH, Sakr W, et al. Phase II randomized clinical trial of lycopene supplementation before radical prostatectomy. Cancer Epidemiol Biomarkers Prev 2001; 10(8): 861-868.

[23] https://blog.cellsignal.com/hallmarks-of-cancer-sustainingproliferative-signaling

[24] Fujiki H, Watanabe T, Sueoka E, Rawangkan A, Suganuma M. Cancer Prevention with Green Tea and Its Principal Constituent, EGCG: from Early Investigations to Current Focus on Human Cancer Stem Cells. Mol Cells 2018; 41(2): 73-82.

[25] Green Tea Extracts for the Prevention of Metachronous Colorectal Adenomas: A Pilot Study Masahito Shimizu,1 Yasushi Fukutomi,2 Mitsuo Ninomiya,3 Kazuo Nagura,4 Tomohiro Kato, Cancer Epidemiol Biomarkers Prev 2008; 17(11). November 2008, American Association for Cancer Research. https://doi.org/10.1158/1055-9965.EPI-08-0528

[26] Gupta SC, Patchva S, Aggarwal BB. Therapeutic roles of curcumin: lessons learned from clinical trials. AAPS J 2013; 15(1): 195-218.

https://doi.org/10.1208/s12248-012-9432-8

[27] Prasad S, Tyagi AK, Aggarwal BB. Recent developments in delivery, bioavailability, absorption and metabolism of curcumin: the golden pigment from golden spice. Cancer Res Treat 2014; 46(1): 2-18. https://doi.org/10.4143/crt.2014.46.1.2

[28] Božović A, Mandušić $V$, Todorović L, Krajnović M. Estrogen Receptor Beta: The Promising Biomarker and Potential Target in Metastases; IJMS 2021; $(22,4)$. https://doi.org/10.3390/ijms22041656

[29] Lazarevic B, Boezelijn G, Diep LM, et al. Efficacy and safety of short-term genistein intervention in patients with localized prostate cancer prior to radical prostatectomy: a randomized, placebo-controlled, double-blind Phase 2 clinical trial. Nutr Cancer 2011; 63(6): 889-898.

https://doi.org/10.1080/01635581.2011.582221

[30] Kang X, Zhang Q, Wang S, Huang X, Jin S. Effect of soy isoflavones on breast cancer recurrence and death for patients receiving adjuvant endocrine therapy. CMAJ 2010; 182(17): 1857-1862.

https://doi.org/10.1503/cmaj.091298

[31] Gleason CE, Carlsson CM, Barnet $\mathrm{JH}$, et al. A preliminary study of the safety, feasibility and cognitive efficacy of soy isoflavone supplements in older men and women. Age Ageing 2009; 38(1): 86-93. https://doi.org/10.1093/ageing/afn227

[32] Block KI, Gyllenhaal C, Lowe L, et al. Designing a broadspectrum integrative approach for cancer prevention and treatment. Semin Cancer Biol 2015; 35(Suppl): S276-S304.
[33] Liu J, Sun Y, Cheng M, et al. Improving Oral Bioavailability of Luteolin Nanocrystals by Surface Modification of Sodium Dodecyl Sulfate. AAPS PharmSciTech 2021; 22: 133. https://doi.org/10.1208/s12249-021-02012-y

[34] Imran $M$, Rauf $A$, Abu-Izneid $T$, Nadeem $M$, Shariati MA, Khan IA, Imran A, Orhan IE, Rizwan M, Atif M, Gondal TA, Mubarak MS. Luteolin, a flavonoid, as an anticancer agent: A review; Biomedicine \& Pharmacotherapy 2019; 112: 108612. https://doi.org/10.1016/j.biopha.2019.108612

[35] Cutando A, López-Valverde A, Arias-Santiago S, De Vicente J, De Diego RG. Role of Melatonin in Cancer Treatment. Anticancer Research 2012; 32(7): 2747-2753.

[36] Yan X, Qi M, Li P, Zhan Y, Shao H. Apigenin in cancer therapy: anti-cancer effects and mechanisms of action. Cell Biosci 2017; 7: 50. https://doi.org/10.1186/s13578-017-0179-x

[37] Milad A, Reza BM, Zahra B, Khandan I, Ali Z, Pooyan M, Haroon K, Samaneh M, Maryam D, Hamed M. Apigenin as Tumor Suppressor in Cancers: Biotherapeutic Activity, Nanodelivery, and Mechanisms With Emphasis on Pancreatic Cancer. Frontiers in Chemistry 2020; 8: 829. https://doi.org/10.3389/fchem.2020.00829

[38] Lall RK, Adhami VM, Mukhtar H. Dietary flavonoid fisetin for cancer prevention and treatment. Mol Nutr Food Res 2016; 60(6): 1396-1405. https://doi.org/10.1002/mnfr.201600025

[39] Farsad-Naeimi A, Alizadeh M, Esfahani A, Aminabad ED. Effect of fisetin supplementation on inflammatory factors and matrix metalloproteinase enzymes in colorectal cancer patients. Food Funct 2018; 9(4): 2025-2031. https://doi.org/10.1039/C7FO01898C

[40] Maher P. How fisetin reduces the impact of age and disease on CNS function. Front Biosci (Schol Ed) 2015; 7: 58-82. https://doi.org/10.2741/s425

[41] Ashrafian L, Sukhikh G, Kiselev V, et al. Double-blind randomized placebo-controlled multicenter clinical trial (phase IIa) on diindolylmethane's efficacy and safety in the treatment of CIN: implications for cervical cancer prevention. EPMA J 2015; 6: 25.

https://doi.org/10.1186/s13167-015-0048-9

[42] Thomson CA, Ho E, Strom MB. Chemopreventive properties of 3,3'-diindolylmethane in breast cancer: evidence from experimental and human studies. Nutr Rev 2016; 74(7): 432443.

https://doi.org/10.1093/nutrit/nuw010

[43] Vigushin DM, Poon GK, Boddy A, et al. Phase I and pharmacokinetic study of D-limonene in patients with advanced cancer. Cancer Research Campaign Phase I/II Clinical Trials Committee. Cancer Chemother Pharmacol 1998; 42(2): 111-117.

https://doi.org/10.1007/s002800050793

[44] Hakim IA, Harris RB, Ritenbaugh C. Citrus peel use is associated with reduced risk of squamous cell carcinoma of the skin. Nutr Cancer 2000; 37(2): 161-168. https://doi.org/10.1207/S15327914NC372_7

[45] de Groot S, Pijl H, van der Hoeven JJM, Kroep JR. Effects of short-term fasting on cancer treatment. J Exp Clin Cancer Res 2019; 38(1): 209. https://doi.org/10.1186/s13046-019-1189-9

[46] Lin BW, Gong CC, Song HF, Cui YY. Effects of anthocyanins on the prevention and treatment of cancer. $\mathrm{Br} \mathrm{J}$ Pharmacol 2017; 174(11): 1226-1243. https://doi.org/10.1111/bph.13627

[47] Pan P, Skaer CW, Stirdivant SM, et al. Beneficial Regulation of Metabolic Profiles by Black Raspberries in Human Colorectal Cancer Patients. Cancer Prev Res (Phila) 2015; 8(8): 743-750. https://doi.org/10.1158/1940-6207.CAPR-15-0065

[48] Janakiram NB, Mohammed A, Rao CV. Sea Cucumbers Metabolites as Potent Anti-Cancer Agents. Marine Drugs 2015; 13(5): 2909-2923. https://doi.org/10.3390/md13052909 
[49] Desai AV, Lu M, Marcus S, Saran A, Malankar A, Mazumder A. A Phase II Trial of TBL12 Sea Cucumber Extract in Patients with Untreated Asymptomatic Myeloma. Blood 2014; 124(21): 5733.

https://doi.org/10.1182/blood.V124.21.5733.5733

[50] Hussain H, Ali I, Wang D, Hakkim FL, Westermann B, Rashan L, Ahmed I, Green IR. Boswellic acids: privileged structures to develop lead compounds for anticancer drug discovery. Expert Opinion on Drug Discovery 2021; 16(8): 851-867. https://doi.org/10.1080/17460441.2021.1892640

[51] Kirste S, Treier M, Wehrle SJ, et al. Boswellia serrata acts on cerebral edema in patients irradiated for brain tumors: a prospective, randomized, placebo-controlled, double-blind pilot trial. Cancer 2011; 117(16): 3788-3795. https://doi.org/10.1002/cncr.25945

[52] Xie G, Dong H, Liang Y, Ham JD, Rizwan R, Chen J. CARNK cells: A promising cellular immunotherapy for cancer. EBioMedicine 2020; 59: 102975. https://doi.org/10.1016/j.ebiom.2020.102975

[53] Darel MB, Valérie D, Denis M. Allogeneic CAR T Cells: An Alternative to Overcome Challenges of CAR T Cell Therapy in Glioblastoma. Frontiers in Immunology 2021; 12: 506. https://doi.org/10.3389/fimmu.2021.640082

[54] McKee DL, Lodhi MS, Mansoor N. The Influence of Tumor Microenvironment on Tumor Progression; and Anticancer Therapies. Journal of Cancer Research Updates 2020; 9(1): 75-81. https://doi.org/10.30683/1929-2279.2020.09.08

[55] Eliza WL, Fai CK, Chung LP. Efficacy of Yun Zhi (Coriolus versicolor) on survival in cancer patients: systematic review and meta-analysis. Recent Pat Inflamm Allergy Drug Discov 2012; 6(1): 78-87. https://doi.org/10.2174/187221312798889310

[56] McCulloch M, See C, Shu XJ, et al. Astragalus-based Chinese herbs and platinum-based chemotherapy for advanced non-small-cell lung cancer: meta-analysis of randomized trials. J Clin Oncol 2006; 24(3): 419-430. https://doi.org/10.1200/JCO.2005.03.6392

[57] Wu P, Dugoua JJ, Eyawo O, Mills EJ. Traditional Chinese Medicines in the treatment of hepatocellular cancers: a systematic review and meta-analysis. J Exp Clin Cancer Res 2009; 28(1): 112.

https://doi.org/10.1186/1756-9966-28-112

[58] Fleischer T, Chang TT, Chiang JH, Sun MF, Yen HR. Improved Survival With Integration of Chinese Herbal Medicine Therapy in Patients With Acute Myeloid Leukemia: A Nationwide Population-Based Cohort Study. Integr Cancer Ther 2017; 16(2): 156-164. https://doi.org/10.1177/1534735416664171

[59] Dhawan DK, Chadha VD. Zinc: a promising agent in dietary chemoprevention of cancer. Indian J Med Res 2010; 132(6): 676-682.

[60] Wu X, Tang J, Xie M. Serum and hair zinc levels in breast cancer: a meta-analysis. Sci Rep 2015; 5: 12249. https://doi.org/10.1038/srep12249

[61] Tompkins TA, Renard NE, Kiuchi A. Clinical evaluation of the bioavailability of zinc-enriched yeast and zinc gluconate in healthy volunteers. Biol Trace Elem Res 2007; 120(1-3): 2835.

https://doi.org/10.1007/s12011-007-0072-2

[62] Zhang S-Q, Yu X-F, Zhang H-B, Peng N, Chen Z-X, Cheng $\mathrm{Q}$, Zhang $\mathrm{X}-\mathrm{L}$, Cheng S-H, Zhang Y. Comparison of the Oral Absorption, Distribution, Excretion, and Bioavailability of Zinc Sulfate, Zinc Gluconate, and Zinc-Enriched Yeast in Rats. Mol Nut Food Research 2018; $(62,7)$. https://doi.org/10.1002/mnfr.201700981

[63] Ribeiro SMF, Braga CBM, Peria FM, Martinez EZ, Rocha JJRD, Cunha SFC. Effects of zinc supplementation on fatigue and quality of life in patients with colorectal cancer. Einstein (Sao Paulo) 2017; 15(1): 24-28.

https://doi.org/10.1590/s1679-45082017a03830

[64] Lin YS, Lin LC, Lin SW. Effects of zinc supplementation on the survival of patients who received concomitant chemotherapy and radiotherapy for advanced nasopharyngeal carcinoma: follow-up of a double-blind randomized study with subgroup analysis. Laryngoscope 2009; 119(7): 1348-1352.

https://doi.org/10.1002/lary.20524

[65] Jin $H$, Jin $X$, Cao B, Wang W. Berberine affects osteosarcoma via downregulating the caspase-1/IL-1 $\beta$ signaling axis. Oncol Rep 2017; 37(2): 729-736.

https://doi.org/10.3892/or.2016.5327

[66] Hou D, Xu G, Zhang C, et al. Berberine induces oxidative DNA damage and impairs homologous recombination repair in ovarian cancer cells to confer increased sensitivity to PARP inhibition. Cell Death Dis 2017; 8: e3070.

https://doi.org/10.1038/cddis.2017.471

[67] Thomas A, Kamble S, Deshkar S, Kothapalli L, Chitlange S. Bioavailability of berberine: Challenges and solutions. İstanbul Journal of Pharmacy 2021; 51(1): 141-153. https://doi.org/10.26650/IstanbulJPharm.2020.0056

[68] Girisa S, Shabnam B, Monisha J, et al. Potential of Zerumbone as an Anti-Cancer Agent. Molecules 2019; 24(4): 734. https://doi.org/10.3390/molecules24040734

[69] Prasad S, Tyagi AK. Ginger and Its Constituents: Role in Prevention and Treatment of Gastrointestinal Cancer. Gastroenterology Research and Practice Volume 2015; Article ID 142979, 11 pages. https://doi.org/10.1155/2015/142979

[70] Ting H, Deep G, Agarwal R. Molecular mechanisms of silibinin-mediated cancer chemoprevention with major emphasis on prostate cancer. AAPS J 2013; 15(3): 707-716. https://doi.org/10.1208/s12248-013-9486-2

[71] Mastron, Jeanetta, Siveen, Kodappully, Sethi, Gautam, Bishayee, Anupam. Silymarin and hepatocellular carcinoma: A systematic, comprehensive, and critical review. Anticancer Drugs 2015; 26. https://doi.org/10.1097/CAD.0000000000000211

[72] Ladas EJ, Kroll DJ, Oberlies $\mathrm{NH}$, et al. A randomized, controlled, double-blind, pilot study of milk thistle for the treatment of hepatotoxicity in childhood acute lymphoblastic leukemia (ALL). Cancer 2010; 116(2): 506-513. https://doi.org/10.1002/cncr.24723

[73] Vidlar A, Vostalova J, Ulrichova J, et al. The safety and efficacy of a silymarin and selenium combination in men after radical prostatectomy - a six month placebo-controlled double-blind clinical trial. Biomed Pap Med Fac Univ Palacky Olomouc Czech Repub 2010; 154(3): 239-244. https://doi.org/10.5507/bp.2010.036

[74] Elyasi S, Hosseini S, Niazi Moghadam MR, Aledavood SA, Karimi G. Effect of Oral Silymarin Administration on Prevention of Radiotherapy Induced Mucositis: A Randomized, Double-Blinded, Placebo-Controlled Clinical Trial. Phytother Res 2016; 30(11): 1879-1885. https://doi.org/10.1002/ptr.5704

[75] Liu H, Dong $Y$, Gao $Y$, et al. The Fascinating Effects of Baicalein on Cancer: A Review. Int J Mol Sci 2016; 17(10): 1681. https://doi.org/10.3390/ijms17101681

[76] A Phase I/II, Multi-Center, Open-Label, Dose-Escalation, Safety and Efficacy Study of PHY906 Plus Capecitabine in Patients With Advanced Pancreatic Carcinoma; ICH GCP; US Clinical Trials Registry; Clinical Trials Nct Page https://ichgcp.net/clinical-trials-registry/NCT00411762.

[77] Xu P, Zhou H, Li YZ, et al. Baicalein Enhances the Oral Bioavailability and Hepatoprotective Effects of Silybin Through the Inhibition of Efflux Transporters BCRP and MRP2. Front Pharmacol 2018; 9: 1115. https://doi.org/10.3389/fphar.2018.01115 
[78] Imran M, Salehi B, Sharifi-Rad J, et al. Kaempferol: A Key Emphasis to Its Anticancer Potential. Molecules 2019; 24(12): 2277.

https://doi.org/10.3390/molecules 24122277

[79] https://www.cancer.gov/news-events/cancer-currentsblog/2020/oligometastatic-cancer-directly-treating-cancermetastases

[80] Jiang WG, Sanders AJ, Katoh M, Ungefroren $\mathrm{H}$, et al. Tissue invasion and metastasis: Molecular, biological and clinical perspectives. Seminars in Cancer Biology 2015; (35, Supplement): S244-S275.

https://doi.org/10.1016/j.semcancer.2015.03.008

[81] Kapoor R, Huang YS. Gamma linolenic acid: an antiinflammatory omega-6 fatty acid. Curr Pharm Biotechnol 2006; 7(6): 531-534. https://doi.org/10.2174/138920106779116874

[82] Jiang WG, Hiscox S, Hallett MB, Horrobin DF, Mansel RE, Puntis MCA. Regulation of the Expression of E-Cadherin on Human Cancer Cells by y-Linolenic Acid (GLA). Cancer Res 1995; 55(21): 5043-504.

[83] Jiang WG, Singhrao SK, Hiscox S, et al. Regulation of desmosomal cell adhesion in human tumour cells by polyunsaturated fatty acids. Clin Exp Metastasis 1997; 15(6): 593-602.

https://doi.org/10.1023/A:1018435229087

[84] Das UN, Prasad VV, Reddy DR. Local application of gammalinolenic acid in the treatment of human gliomas. Cancer Lett 1995; 94(2): 147-155.

https://doi.org/10.1016/0304-3835(95)03844-M

[85] Xu Y, Qian SY. Anti-cancer activities of $\omega-6$ polyunsaturated fatty acids. Biomed J 2014; 37(3): 112-119. https://doi.org/10.4103/2319-4170.131378

[86] van der Merwe CF, Booyens J, Joubert HF, van der Merwe $\mathrm{CA}$. The effect of gamma-linolenic acid, an in vitro cytostatic substance contained in evening primrose oil, on primary liver cancer. A double-blind placebo controlled trial. Prostaglandins Leukot Essent Fatty Acids 1990; 40(3): 199202.

\section{https://doi.org/10.1016/0952-3278(90)90098-6}

[87] Rose DP, Rayburn J, Hatala MA, Connolly JM. Effects of dietary fish oil on fatty acids and eicosanoids in metastasizing human breast cancer cells. Nutr Cancer 1994; 22(2): 131-141.

https://doi.org/10.1080/01635589409514338

[88] Kinoshita K, Noguchi M, Tanaka M. Effects of linoleic acid, eicosapentaenoic acid, and docosahexaenoic acid on the growth and metastasis of MM48 mammary tumor transplants in mice. International Journal of Oncology 1996; 8: 575-581. https://doi.org/10.3892/ijo.8.3.575

[89] Suzuki I, ligo M, Ishikawa C, Kuhara T, Asamoto M, Kunimoto T, Moore MA, Yazawa K, Araki E, Tsuda H. Inhibitory effects of oleic and docosahexaenoic acids on lung metastasis by colon-carcinoma- 26 cells are associated with reduced matrix metalloproteinase- 2 and -9 activities. Int $\mathrm{J}$ Cancer 1997; 73: 607-612.

https://doi.org/10.1002/(SICl)1097-

0215(19971114)73:4<607::AID-IJC24>3.0.CO;2-4

[90] Yam, Daniel \& Peled, Alpha \& Shinitzky, Meir. Suppression of tumor growth and metastasis by dietary fish oil combined with vitamins $E$ and $C$ and cisplatin. Cancer Chemotherapy and Pharmacology 2001; 47: 34-40. https://doi.org/10.1007/s002800000205

[91] Bougnoux P, Hajjaji N, Ferrasson MN, Giraudeau B, Couet $C$, Le Floch O. Improving outcome of chemotherapy of metastatic breast cancer by docosahexaenoic acid: a phase II trial. Br J Cancer 2009; 101(12): 1978-1985. https://doi.org/10.1038/sj.bjc.6605441

[92] Merendino N, Costantini L, Manzi L, Molinari R, D'Eliseo D, Velotti F. Dietary $\omega-3$ Polyunsaturated Fatty Acid DHA: A
Potential Adjuvant in the Treatment of Cancer 2013; Article ID 310186.

https://doi.org/10.1155/2013/310186

[93] Murphy RA, Mourtzakis M, Chu QS, Baracos VE, Reiman T, Mazurak VC. Nutritional intervention with fish oil provides a benefit over standard of care for weight and skeletal muscle mass in patients with nonsmall cell lung cancer receiving chemotherapy. Cancer 2011; 117(8): 1775-1782. https://doi.org/10.1002/cncr.25709

[94] Camargo Cde Q, Mocellin MC, Pastore Silva Jde A, Fabre ME, Nunes EA, Trindade EB. Fish oil supplementation during chemotherapy increases posterior time to tumor progression in colorectal cancer. Nutr Cancer 2016; 68(1): 70-76. https://doi.org/10.1080/01635581.2016.1115097

[95] Trabal J, Leyes P, Forga M, Maurel J. Potential usefulness of an EPA-enriched nutritional supplement on chemotherapy tolerability in cancer patients without overt malnutrition. Nutr Hosp 2010; 25(5): 736-740.

[96] Murphy RA, Mourtzakis M, Chu QS, Baracos VE, Reiman T, Mazurak VC. Supplementation with fish oil increases first-line chemotherapy efficacy in patients with advanced nonsmall cell lung cancer. Cancer 2011; 117(16): 3774-3780. https://doi.org/10.1002/cncr.25933

[97] Weseler AR, Bast A. Masquelier's grape seed extract: from basic flavonoid research to a well-characterized food supplement with health benefits. Nutr J 2017; 16(1): 5 . https://doi.org/10.1186/s12937-016-0218-1

[98] Nandakumar V, Singh T, Katiyar SK. Multi-targeted prevention and therapy of cancer by proanthocyanidins. Cancer Lett 2008; 269(2): 378-387. https://doi.org/10.1016/j.canlet.2008.03.049

[99] Ravindranathan P, Pasham D, Balaji U, et al. Mechanistic insights into anticancer properties of oligomeric proanthocyanidins from grape seeds in colorectal cancer. Carcinogenesis 2018; 39(6): 767-777.

https://doi.org/10.1093/carcin/bgy034

[100] Kim HY, Kim YM, Hong S. Astaxanthin suppresses the metastasis of colon cancer by inhibiting the MYC-mediated downregulation of microRNA-29a-3p and microRNA-200a. Sci Rep 2019; 9: 9457. https://doi.org/10.1038/s41598-019-45924-3

[101] McCall B, McPartland CK, Moore R, Frank-Kamenetskii A Booth BW. Effects of Astaxanthin on the Proliferation and Migration of Breast Cancer Cells In vitro. Antioxidants (Basel) 2018; 7(10): 135. https://doi.org/10.3390/antiox7100135

[102] Andrea DY, Javiera G, Andrés AM, Pablo G, Cristian A. Therapeutic uses of natural astaxanthin: An evidence-based review focused on human clinical trials. Pharmacological Research 2021; 166: 105479.

https://doi.org/10.1016/j.phrs.2021.105479

[103] Li F, Zhou K, Gao L, et al. Radiation induces the generation of cancer stem cells: A novel mechanism for cancer radioresistance. Oncol Lett 2016; 12(5): 3059-3065. https://doi.org/10.3892/ol.2016.5124

[104] Li F, Zhou K, Gao L, et al. Radiation induces the generation of cancer stem cells: A novel mechanism for cancer radioresistance. Oncol Lett 2016; 12(5): 3059-3065. https://doi.org/10.3892/ol.2016.5124

[105] Naujokat C, McKee DL. The "Big Five" Phytochemicals Targeting Cancer Stem Cells: Curcumin, EGCG, Sulforaphane, Resveratrol and Genistein. Curr Med Chem. 2021; 28(22): 4321-4342. https://doi.org/10.2174/0929867327666200228110738

[106] Gupta PB, Onder TT, Jiang G, et al. Identification of selective inhibitors of cancer stem cells by high-throughput screening. Cell 2009; 138(4): 645-659.

https://doi.org/10.1016/j.cell.2009.06.034 
[107] Taylor WF, Jabbarzadeh E. The use of natural products to target cancer stem cells. Am J Cancer Res 2017; 7(7): 15881605.

[108] Chamberlin SR, Blucher A, Wu G, Shinto L, Choonoo G, Kulesz-Martin M, McWeeney $S$. Natural Product Target Network Reveals Potential for Cancer Combination Therapies. Frontiers in Pharmacology 2019; 10: 557. https://doi.org/10.3389/fphar.2019.00557
[109] Mehta D, Uber R, Ingle T, et al. Study of pharmacogenomic information in FDA-approved drug labeling to facilitate application of precision medicine. Drug Discovery Today 2020; 25(5): 813-820.

https://doi.org/10.1016/j.drudis.2020.01.023

Received on 10-11-2021

Accepted on 25-11-2021

Published on 20-12-2021

https://doi.org/10.30683/1929-2279.2021.10.08

(c) 2021 McKee and Lodhi; Licensee Neoplasia Research.

This is an open access article licensed under the terms of the Creative Commons Attribution Non-Commercial License (http://creativecommons.org/licenses/by-nc/3.0/) which permits unrestricted, non-commercial use, distribution and reproduction in any medium, provided the work is properly cited. 